\title{
The Instability in the Dimensions of Spline Spaces Over $T$-Meshes with Nested $T$-Cycles
}

\author{
Chongjun $\mathrm{Li}^{*}$ and Pengxiao Wang \\ School of Mathematical Sciences, Dalian University of Technology, \\ Dalian 116024, China \\ Received 21 September 2017; Accepted (in revised version) 8 March 2018
}

\begin{abstract}
This paper studies on the dimensions of spline spaces over some given $T$ meshes. Using the smoothing cofactor-conformality method, we study the instability in the dimensions of the spline spaces over $T$-meshes with 2-nested and 3-nested $T$ cycles. We define a singularity factor of each simple $T$-cycle, the instability and the structure's degeneration are associated with the singularity factors. In order to get a stable dimension formula over $T$-mesh with a $N$-nested $T$-cycle, a constraint on the $T$-mesh is introduced. Finally, a possible degeneration for a case of parallel $T$-cycles is illustrated.
\end{abstract}

AMS subject classifications: $65 \mathrm{D} 07$

Key words: Spline space, smoothing cofactor-conformality method, instability in the dimension, $T$-mesh, $T$-cycle.

\section{Introduction}

As an industry standard, the Non-Uniform Rational B-spline (NURBS) is commonly used in computer-aided design (CAD), manufacturing (CAM), and engineering (CAE). However, NURBS models suffer from a major weakness that they contain a large number of superfluous control points $[1,2]$. To overcome this limitation, the $T$-spline, a pointbased spline defined on $T$-mesh, was invented by Sederberg et al. [1]. It facilitates local refinement and makes the representation of free-form surfaces more flexible. In addition, a class of $T$-splines called Analysis-suitable $T$-splines has been proposed, for which basis functions are linearly independent and form a partition of unity [3,4]. Scott et al. [4] also gave a greedy algorithm to achieve the local refinement for Analysis-suitable $T$-splines.

Besides, there is another way to obtain local modification on the tensor-product Bsplines over rectangular meshes. The spline space over a $T$-mesh, denoted by $S(m, n, \alpha, \beta$, $\mathscr{T})$, was firstly introduced in [5], which is a bi-degree $(m, n)$ piecewise polynomial spline space over $T$-mesh $\mathscr{T}$ with smoothness order $\alpha$ along $x$ direction and $\beta$ in $y$ direction. By using the B-net method, Deng et al. [5] calculated the dimension of spline

${ }^{*}$ Corresponding author. Email addresses: chongjun@dlut.edu.cn (C. J. Li), wangpengxiao@mail.dlut. edu. cn (P. X. Wang) 
space $S(m, n, \alpha, \beta, \mathscr{T})$ with constraints of $m \geq 2 \alpha+1$ and $n \geq 2 \beta+1$ when the $T$-mesh has no $T$-cycle. Huang et al. [6] derived an equivalent dimension formula in a different form by the smoothing cofactor-conformality method $[7,8]$. Li et al. $[9,10]$ improved the dimension formula in the same spline space by using the smoothing cofactor-conformality method with a constraint depending on the order of the smoothness, the degree of the spline functions and the structure of the T-mesh as well. Further, Li et al. [11] discussed the stability of the dimensions of general spline spaces based on the analysis of the conformality condition at one interior vertex. Mourrain [12] defined the weighted $T$-meshes, over which the dimension could be computed in an explicit formula by using the homological techniques. Li et al. [13] introduced a class of T-meshes, diagonalizable T-meshes, over which the dimensions of spline spaces are stable. Wang et al. [14] proposed a de Boor like algorithm to evaluate PHT-splines-polynomial splines over hierarchical $T$-meshes with stable dimensions. However, the case of instable dimension of the spline space over $T$-mesh was discussed insufficiently.

As we known, for the Morgan-Scott triangulation, the dimension of spline space depends on not only the topological information of the partition but also the geometry structure of the partition [15]. Recently, Li et al. [16] and Berdinsky et al. [17] found the instability of the dimensions of spline spaces over some certain $T$-meshes. In 2015, Guo et al. [18] discovered the instability of the dimension for the $T$-mesh with an independent simple $T$-cycle. However, if several $T$-cycles are connected to each other or to say dependent to each other, it is difficult to determine the associated dimension. In this paper, we study on the dimensions of spline spaces over some given $T$-meshes with nested $T$-cycles and parallel $T$-cycles which are composed of several connected $T$-cycles. We find some very interesting results on the dimensions of the corresponding spline spaces. For the cases of 2-nested and 3-nested $T$-cycles, the instability is associated with corresponding singularity factor of each $T$-cycle. In addition, we give a stable dimension formula over $T$-mesh with a $N$-nested $T$-cycle by a constraint on the $T$-mesh.

The remainder of this paper is organized as follows. We review on some definitions and notations regarding the $T$-meshes and give the definition of $N$-nested $T$-cycle in Section 2. In Section 3, we discuss the instability on the dimensions of spline spaces over $T$-meshes with 2-nested and 3-nested $T$-cycles respectively. Moreover, we show that the instability of the dimension and the degeneration of the corresponding structure of the $T$-mesh are related to a geometric condition which is defined as singularity factor and we introduce a constraint on the $T$-mesh in order to obtain a stable dimension formula for $T$-mesh with a $N$-nested $T$-cycle. In Section 4, a case of parallel $T$-cycles on the degeneration of $T$-mesh is presented. Finally, conclusions are drawn in the last section.

\section{Preliminaries}

In this section, we briefly review some definitions and notations of $T$-mesh as in [9], then give some prior work on the instability in the dimension of spline space.

A T-mesh is a modified rectangular grid that allows $T$-junctions arising from $T$-spline. For a given $T$-mesh as shown in Fig. 1(a), its interior mesh segments are divided into three 


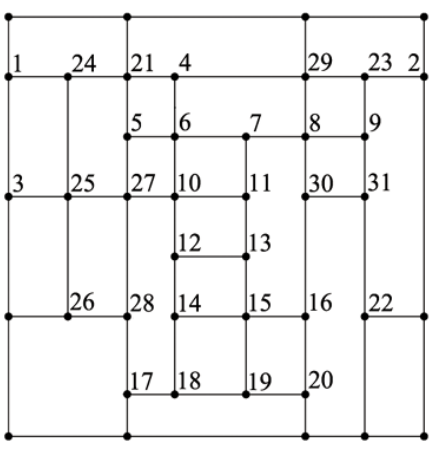

(a) $T$-mesh

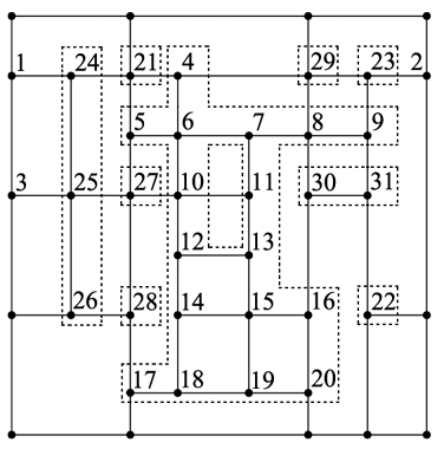

(b) $T$-connected components

Figure 1: A $T$-mesh and its $T$-connected components.

kinds as follows:

a) cross-cut: both of its endpoints lie on the boundary of the mesh, e.g. $\overline{v_{1} v_{2}}$ in Fig. 1(a);

b) ray: only one of its endpoints lies on the boundary of the mesh, e.g. $\overline{v_{3} v_{11}}$ in Fig. 1(a);

c) $T$-segment: both of its endpoints are interior vertices, e.g. $\overline{v_{4} v_{18}}$ in Fig. 1(a).

According to the three kinds of mesh segments above, interior vertices are also divided into three kinds:

1) free-vertex: the intersection point of cross-cuts or rays, e.g, $v_{21}, v_{22}, v_{27}$ in Fig. 1(a).

2) mono-vertex: the intersection point of one $T$-segment and one cross-cut or one ray, e.g. $v_{5}, v_{9}, v_{16}$ in Fig. 1(a).

3) multi-vertex: the intersection point of two T-segments, e.g, $v_{6}, v_{15}, v_{18}$ in Fig. 1(a). In this case, we say the two $T$-segments are $T$-connected, e.g, $\overline{v_{5} v_{9}}$ and $\overline{v_{4} v_{18}}$ are $T$ connected at $v_{6}$.

For a given $T$-mesh $\mathscr{T}$, the spline space is denoted by $S(m, n, \alpha, \beta, \mathscr{T})$. By the results in [9], for each interior vertex $v_{i} \in \mathscr{T}$, there exists a conformality factor $d_{i}(x, y) \in$ $\mathbb{P}_{m-\alpha-1, n-\beta-1}$, where $\mathbb{P}_{m-\alpha-1, n-\beta-1}$ is the bi-degree polynomial space. The dimension of spline space is determined by the degrees of freedom of all the conformality factors. However, those factors need satisfying some conformality conditions along each T-segment. For example, denote $x_{5}, x_{6}, x_{7}, x_{8}, x_{9}$ by the $x$-coordinates of the vertices $v_{5}, v_{6}, v_{7}, v_{8}, v_{9}$ on the same $T$-segment in Fig. 1(a). The corresponding conformality condition along this horizontal $T$-segment is

$$
d_{5}(x, y)\left(x-x_{5}\right)^{\alpha+1}+d_{6}(x, y)\left(x-x_{6}\right)^{\alpha+1}+\cdots+d_{9}(x, y)\left(x-x_{9}\right)^{\alpha+1} \equiv 0 .
$$

A $T$-connected component is defined as the union of all $T$-connected $T$-segments and their vertices since the corresponding conformality conditions are coupled. E.g. the union 


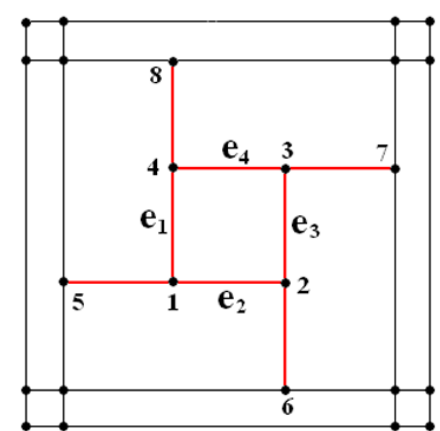

(a) A T-cycle

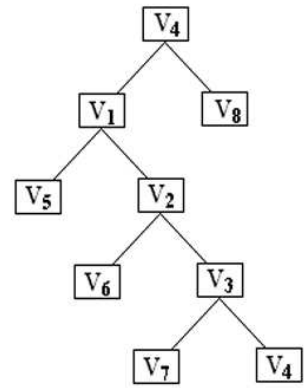

(b) The binary tree of (a)

Figure 2: A T-cycle with its binary tree.

of $\overline{v_{4} v_{18}}, \overline{v_{5} v_{9}}, \overline{v_{7} v_{19}}, \overline{v_{12} v_{13}}, \overline{v_{14} v_{16}}, \overline{v_{17} v_{20}}$ in Fig. 1(b), which must be regarded as a local structure. Then the corresponding conformality conditions for different $T$-connected components can be solved individually. By decomposing the $T$-mesh into several $T$-connected components without intersections (as shown in Fig. 1(b)), the dimension formula can be proved in [9].

A $T$-connected component is a connected component with $T$-junctions. If the vertex is an endpoint of one $T$-segment, which lies on one cross-cut or one ray, then we call it as boundary vertex of the $T$-connected component. Referring to [9], a kind of binary tree for a given $T$-connected component is described. Selecting an interior vertex as root, its two child nodes are the two end vertices of the $T$-segment where the root lies. If the node is a boundary vertex of the $T$-connected component, it has no child. Specially, there exists a case that the procedure is a recurrence when one node becomes to be one of its descendants, which is defined by T-cycle as follows.

Definition 2.1 ( $T$-cycle). We build a binary tree structure for a $T$-connected component in a given $T$-mesh. If one node appears to be one of its descendants, then we call the T-connected component as a T-cycle. And we call the boundary vertices of the T-cycle as the leaf nodes of its corresponding binary tree.

As shown in Fig. 2, the $T$-connected component formed by four $T$-segments $e_{1}, e_{2}, e_{3}, e_{4}$ is a $T$-cycle, the leaf nodes $v_{5}, v_{6}, v_{7}, v_{8}$ are boundary vertices of the $T$-cycle.

Definition 2.2 (Simple T-cycle). For a T-cycle including $N T$-segments $e_{1}, \cdots, e_{N}$, by Definition 2.1, if they can be arranged in an order as

$$
e_{1} \rightarrow e_{2} \rightarrow \cdots \rightarrow e_{N-1} \rightarrow e_{N} \rightarrow e_{1},
$$

such that only one of the end vertices of $e_{j}$ is an interior vertex of $e_{j+1}, j=1, \cdots, N$, the other end vertex is not the interior vertex of other T-segments of the T-cycle, where $e_{N+1}=e_{1}$. Then the T-cycle is called a simple T-cycle. As shown in Fig. 3, the T-connected component formed by $e_{1}, e_{2}, \cdots, e_{6}$ is a simple T-cycle. 


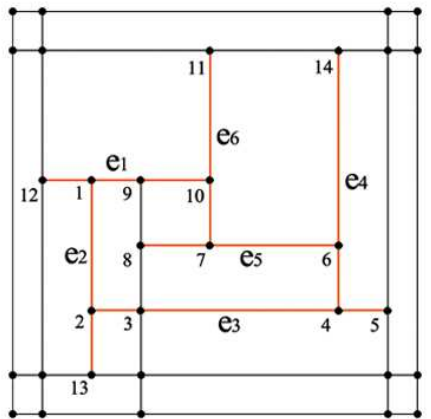

Figure 3: A T-mesh with a simple T-cycle.

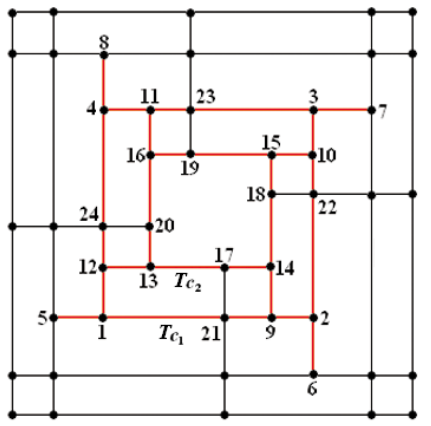

Figure 5: A T-mesh with a 2-nested T-cycle.

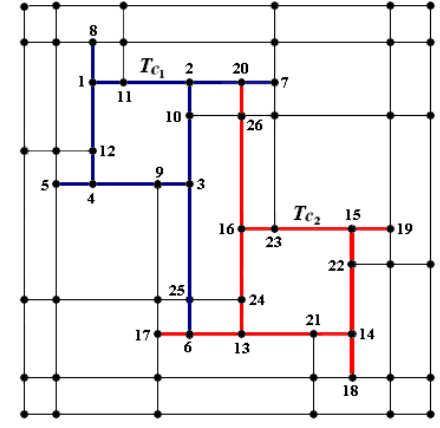

Figure 4: A T-mesh with a pair of parallel Tcycles.

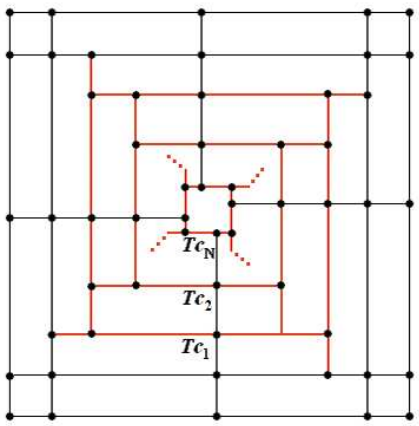

Figure 6: A T-mesh with a $N$-nested T-cycle.

In many cases, there is not only one simple $T$-cycle in a $T$-mesh. Fig. 4 and Fig. 5 are two different cases.

In Fig. 4, there are two simple $T$-cycles $T c_{1}=\left\{\overline{v_{5} v_{3}}, \overline{v_{6} v_{2}}, \overline{v_{7} v_{1}}, \overline{v_{8} v_{4}}\right\}$ and $T c_{2}=\left\{\overline{v_{17} v_{14}}\right.$, $\left.\overline{v_{18} v_{15}}, \overline{v_{19} v_{16}}, \overline{v_{20} v_{13}}\right\}$. A boundary vertex $v_{6}$ of $T c_{1}$ is an interior vertex of $T c_{2}$, and a boundary vertex $v_{20}$ of $T c_{2}$ is an interior vertex of $T c_{1}$. We call this case by a pair of parallel $T$-cycles.

In Fig. 5, there are also two simple $T$-cycles $T c_{1}=\left\{\overline{v_{5} v_{2}}, \overline{v_{6} v_{3}}, \overline{v_{7} v_{4}}, \overline{v_{8} v_{1}}\right\}$ and $T c_{2}=$ $\left\{\overline{v_{12} v_{14}}, \overline{v_{9} v_{15}}, \overline{v_{10} v_{16}}, \overline{v_{11} v_{13}}\right\}$. But in this case, all boundary vertices $v_{9}, v_{10}, v_{11}, v_{12}$ of $T c_{2}$ are interior vertices of $T c_{1}$. We call this case by a 2-nested $T$-cycle. In general, we can define $N$-nested T-cycle as follows.

Definition 2.3 ( $N$-nested T-cycle). We nest $N-1$ simple $T$-cycles $T c_{2}, T c_{3}, \cdots, T c_{N}$ layer by layer inside of a simple $T$-cycle $T c_{1}$, if they can be arranged in an order as

$$
T c_{1} \rightarrow T c_{2} \rightarrow \cdots \rightarrow T c_{N-1} \rightarrow T c_{N}
$$

such that all the boundary vertices of $T c_{i}, i=2, \cdots, N$ are interior vertices of $T c_{i-1}$. Then this structure is called a $N$-nested T-cycle as showed in Fig. 6.

For arbitrary T-mesh, the structure may be very complicated, we only discuss the above two cases in this paper. 


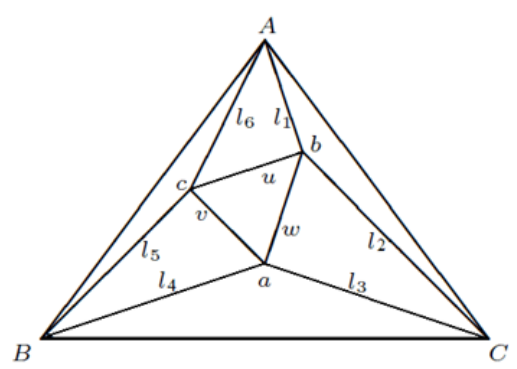

Figure 7: The Morgan-Scott's partition $\Delta_{M S}$.

\section{The dimensions of spline spaces over proper $T$-meshes with $N$-nested $T$-cycles}

\subsection{Improvement on the singularity condition of $\operatorname{dim} S\left(2,2,1,1, \mathscr{T}_{1}\right)$}

Let us review some results on the singularity of space $S_{2}^{1}\left(\Delta_{M S}\right)$ firstly. The dimension of spline space with degree 2 and smoothness order 1 over the triangulation shown in Fig. 5 heavily depends on the geometric property of the partition. Shi [19] and Diener [20] obtained independently that the necessary and sufficient condition of $\operatorname{dim} S_{2}^{1}\left(\Delta_{M S}\right)=7$ is that $A a, B b, C c$ are concurrent. From the algebraic geometry viewpoint, Du [21] obtained equivalent condition: if the six quasi-inner edges are regarded as six points in the projective plane, then they lie on a conic. Obviously, the equivalence of the results obtained by Shi and Du is sustained by Pascal's Theorem: If a hexagon is inscribed in a conic in the project plane, then the opposite sides of the hexagon meet in collinear points.

For the Morgan-Scott triangulation, the dimension of $S_{2}^{1}\left(\Delta_{M S}\right)$ can also be solved via the Generator Basis method by Luo [22]. let

$$
\left\{\begin{array}{l}
l_{1}=\alpha_{1} u+\beta_{1} v \\
l_{2}=\alpha_{2} u+\beta_{2} v
\end{array},\left\{\begin{array} { l } 
{ l _ { 3 } = \alpha _ { 3 } v + \beta _ { 3 } w } \\
{ l _ { 4 } = \alpha _ { 4 } v + \beta _ { 4 } w }
\end{array} \text { and } \left\{\begin{array}{l}
l_{5}=\alpha_{5} w+\beta_{5} u \\
l_{6}=\alpha_{6} w+\beta_{6} u
\end{array}\right.\right. \text {. }\right.
$$

It has the following conclusion in algebraic form:

Theorem 3.1. ([22])The spline space is singular $\left(\operatorname{dim} S_{2}^{1}\left(\Delta_{M S}\right)=7\right)$ if and only if

$$
\frac{\beta_{1} \beta_{2}}{\alpha_{1} \alpha_{2}} \cdot \frac{\beta_{3} \beta_{4}}{\alpha_{3} \alpha_{4}} \cdot \frac{\beta_{5} \beta_{6}}{\alpha_{5} \alpha_{6}}=1
$$

where the value of $\frac{\beta_{1} \beta_{2}}{\alpha_{1} \alpha_{2}} \cdot \frac{\beta_{3} \beta_{4}}{\alpha_{3} \alpha_{4}} \cdot \frac{\beta_{5} \beta_{6}}{\alpha_{5} \alpha_{6}}$ is the characteristic number of a conic.

For the case of $T$-mesh, Guo et al. [18] gave a necessary and sufficient condition of the singularity of $\operatorname{dim} S\left(2,2,1,1, \mathscr{T}_{1}\right)$ over $T$-mesh $\mathscr{T}_{1}$ with a $T$-cycle given in Fig. 8 by using the smoothing cofactor-conformality method as follows.

Theorem 3.2. ([18])The necessary and sufficient condition of singularity of dim $S(2,2,1,1$, $\mathscr{T}_{1}$ ) is $G_{s t}=1$, where $G_{s t}=\frac{s_{5,1} s_{5,4}}{s_{2,1} s_{2,4}} \frac{s_{2,3} s_{2,6}}{s_{5,3} s_{5,6}} \frac{t_{5,1} t_{5,4}}{t_{2,1} t_{2,4}} \frac{t_{2,3} t_{2,6}}{t_{5,3} t_{5,6}}, s_{i, j}=x_{i}-x_{j}, t_{i, j}=y_{i}-y_{j}$. If $G_{s t}=1$, the dimension of $S\left(2,2,1,1, \mathscr{T}_{1}\right)$ is 30 ; otherwise the dimension of $S\left(2,2,1,1, \mathscr{T}_{1}\right)$ is 29 . 


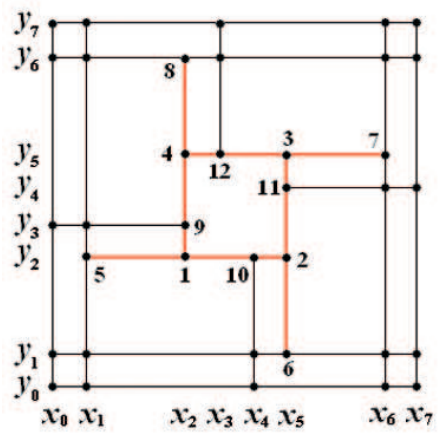

Figure 8: A T-mesh with a T-cycle $\mathscr{T}_{1}$.

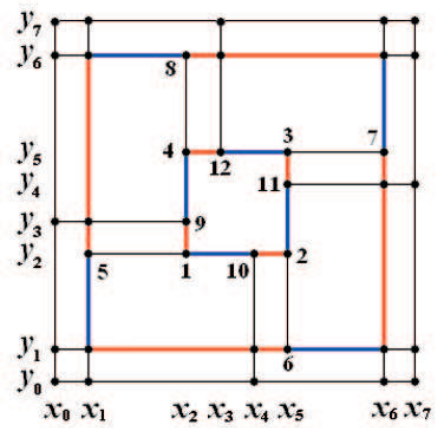

Figure 9: Alternant segments of red and blue color in $\mathscr{T}_{1}$.

Since the instability of the dimension depends on the value of $G_{s t}$, we call the number by the singularity factor of the $T$-cycle. The meaning of the singularity factor can be explained in two viewpoints.

It is easy to verify that the singularity factor $G_{s t}$ is equal to the product of four associated cross ratios on the given points along the $x$-axis and $y$-axis as follows.

$$
G_{s t}=\left(x_{4} x_{3}, x_{5} x_{2}\right) \cdot\left(x_{1} x_{6}, x_{5} x_{2}\right) \cdot\left(y_{4} y_{3}, y_{5} y_{2}\right) \cdot\left(y_{1} y_{6}, y_{5} y_{2}\right),
$$

where $\left(P_{1} P_{2}, P_{3} P_{4}\right)$ is the cross ratio of the points $P_{1}, P_{2}, P_{3}, P_{4}$ and

$$
\left(P_{1} P_{2}, P_{3} P_{4}\right)=\frac{\left(P_{1}-P_{3}\right)\left(P_{2}-P_{4}\right)}{\left(P_{2}-P_{3}\right)\left(P_{1}-P_{4}\right)} .
$$

In addition, it has the following geometric meaning.

Theorem 3.3. The necessary and sufficient condition of the singularity of $\operatorname{dim} S\left(2,2,1,1, \mathscr{T}_{1}\right)$ is that the ratio between the product of 8 red segment lengths and the product of 8 blue segment lengths is 1 in Fig. 9.

Since the red segments and blue segments are alternant in $\mathscr{T}_{1}$, we call the ratio between the product of 8 red segment lengths and the product of 8 blue segment lengths as an 


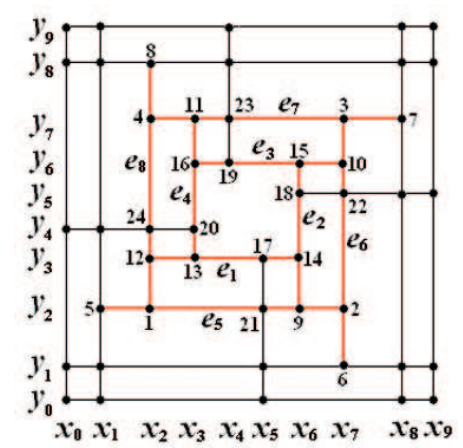

Figure 10: A $T$-mesh with a 2-nested $T$-cycle $\mathscr{T}_{2}$.

alternative product ratio. This geometric condition is the same as singularity factor of the $T$-cycle. In the following, we discuss the case of $T$-mesh with a $N$-nested $T$-cycle as shown in Fig. 6. Similar to Theorem 3.2, there exists an corresponding singularity factor for each simple $T$-cycle, denoted by $a_{i}(i=1, \cdots, N)$. We will see that the dimension of the spline space is instable due to different values of the singularity factors $a_{i}(i=1, \cdots, N)$.

\subsection{The dimension of the spline space on a T-mesh with a 2-nested T-cycle}

Example 3.1. Now we consider the case of one $T$-cycle nested inside of another $T$-cycle as showed in Fig. 10 (same as Fig. 5). Assume that $\mathbf{x}=\left(x_{i}\right)_{i=0}^{9}$ and $\mathbf{y}=\left(y_{i}\right)_{y=0}^{9}$ are two ascending sequences. The two $T$-cycles are $T c_{1}$ and $T c_{2}$, where $T c_{1}=\left\{e_{5}=\overline{v_{5} v_{2}}, e_{6}=\right.$ $\left.\overline{v_{6} v_{3}}, e_{7}=\overline{v_{7} v_{4}}, e_{8}=\overline{v_{8} v_{1}}\right\}, T c_{2}=\left\{e_{1}=\overline{v_{12} v_{14}}, e_{2}=\overline{v_{9} v_{15}}, e_{3}=\overline{v_{10} v_{16}}, e_{4}=\overline{v_{11} v_{13}}\right\}$.

According to Theorem 3.2, the two singularity factors $a_{1}$ and $a_{2}$ corresponding to $T c_{1}$ and $T c_{2}$ are

$$
\begin{aligned}
& a_{1}=\frac{s_{5,7} s_{1,7}}{s_{1,2} s_{2,5}} \frac{s_{2,8} s_{2,4}}{s_{7,8} s_{4,7}} \frac{t_{5,7} t_{1,7}}{t_{1,2} t_{2,5}} \frac{t_{2,8} t_{2,4}}{t_{7,8} t_{4,7}}, \\
& a_{2}=\frac{s_{5,6} s_{2,6}}{s_{2,3} s_{3,5}} \frac{s_{3,7} s_{3,4}}{s_{6,7} s_{4,6}} \frac{t_{5,6} t_{2,6}}{t_{2,3} t_{3,5}} \frac{t_{3,7} t_{3,4}}{t_{6,7} t_{4,6}} .
\end{aligned}
$$

Theorem 3.4. The dimension of spline space $S\left(2,2,1,1, \mathscr{T}_{2}\right)$ over $T$-mesh $\mathscr{T}_{2}$ shown in Fig. 10 is unstable. The T-mesh's degeneration of two different cases are shown in Fig. 11.

$$
\begin{aligned}
& \operatorname{dimS}\left(2,2,1,1, \mathscr{T}_{2}\right) \\
& = \begin{cases}29, & \text { if } \left.a_{1} \neq 1 \text { and } a_{2} \neq 1 \text { (Both } T c_{1} \text { and } T c_{2} \text { are degenerated from } \mathscr{T}_{2}\right), \\
30, & \text { if } a_{1}=1 \text { and for any } a_{2}\left(T c_{2} \text { is degenerated from } \mathscr{T}_{2}\right), \\
30, & \text { if } a_{1} \neq 1 \text { and } a_{2}=1 \text { (None degeneration), }\end{cases}
\end{aligned}
$$

where $a_{1}$ and $a_{2}$ are defined by (3.1a) and (3.1b).

Proof. See Appendix A. 


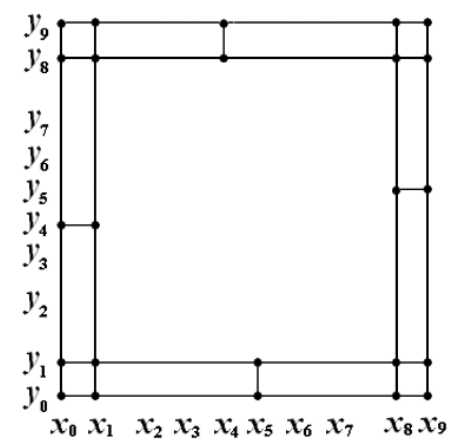

(a) Both of the two T-cycles are degenerated.

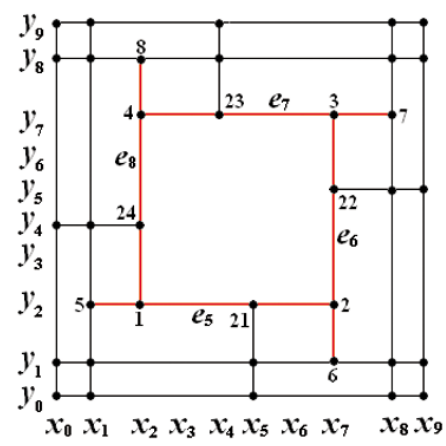

(b) The inner T-cycle $T c_{2}$ is degenerated.

Figure 11: The two degenerations of $\mathrm{T}$-mesh $\mathscr{T}_{2}$.

We see that the dimension and structure's degeneration are related to the value of singularity factor of each $T$-cycle. Fig. 11 shows two different cases on $T$-mesh's degeneration and we give the following examples in different cases.

Remark 3.1. (i) We set $\left(x_{0}, x_{1}, \cdots, x_{9}\right)=\left(-1,0,1,2, \frac{5}{2}, \frac{8}{3}, 3,4,5,6\right)$ and $\left(y_{0}, y_{1}\right.$, $\left.\cdots, y_{9}\right)=\left(-1,0,1,2, \frac{5}{2}, \frac{8}{3}, 3,4,5,6\right)$ in Fig. 10. By computing, $a_{1} \neq 1$ and $a_{2} \neq 1$, hence $\operatorname{dim} S\left(2,2,1,1, \mathscr{T}_{2}\right)=29$ and two $T$-cycles are totally degenerated from $\mathscr{T}_{2}$.

(ii) We set $\left(x_{0}, x_{1}, \cdots, x_{9}\right)=\left(-12,-11,1, \frac{3}{2}, \frac{7}{3}, \frac{8}{3}, 3,4,16,17\right)$ and $\left(y_{0}, y_{1}, \cdots, y_{9}\right)=$ $\left(-12,-11,1, \frac{3}{2}, \frac{7}{3}, \frac{8}{3}, 3,4,16,17\right)$ in Fig. 10. By computing, $a_{1}=1$ and $a_{2} \neq 1$, hence $\operatorname{dim} S\left(2,2,1,1, \mathscr{T}_{2}\right)=30$ and the inner $T$-cycle $T c_{2}$ is degenerated from $\mathscr{T}_{2}$.

(iii) We set $\left(x_{0}, x_{1}, \cdots, x_{9}\right)=\left(-12,-11,1,2, \frac{7}{3}, \frac{8}{3}, 3,4,16,17\right)$ and $\left(y_{0}, y_{1}, \cdots, y_{9}\right)=$ $\left(-12,-11,1,2, \frac{7}{3}, \frac{8}{3}, 3,4,16,17\right)$ in Fig. 10. By computing, $a_{1}=1$ and $a_{2}=1$, hence $\operatorname{dim} S\left(2,2,1,1, \mathscr{T}_{2}\right)=30$ and the inner $T$-cycle $T c_{2}$ is degenerated from $\mathscr{T}_{2}$.

(iv) We set $\left(x_{0}, x_{1}, \cdots, x_{9}\right)=\left(-1,0,1,2, \frac{7}{3}, \frac{8}{3}, 3,4,5,6\right)$ and $\left(y_{0}, y_{1}, \cdots, y_{9}\right)=$ $\left(-1,0,1,2, \frac{7}{3}, \frac{8}{3}, 3,4,5,6\right)$ in Fig. 10. By computing, $a_{1} \neq 1$ and $a_{2}=1$, hence $\operatorname{dim} S\left(2,2,1,1, \mathscr{T}_{2}\right)=30$ and $\mathscr{T}_{2}$ is non-degenerate.

We next provide another case of T-mesh with a 2-nested mixed T-cycle that the dimension of spline space is unstable, which is an extension to the structure of $\mathscr{T}_{2}$.

Example 3.2. In this example, we consider the spline space $S\left(2,2,1,1, \mathscr{T}_{2}^{\prime}\right)$ over the Tmesh $\mathscr{T}_{2}^{\prime}$ with a four-segments' $T$-cycle that nested inside of a simple $T$-cycle with six segments as shown in Fig. 12. And we assume that $\mathbf{x}=\left(x_{i}\right)_{i=0}^{11}$ and $\mathbf{y}=\left(y_{i}\right)_{y=0}^{11}$ are two ascending sequences. 


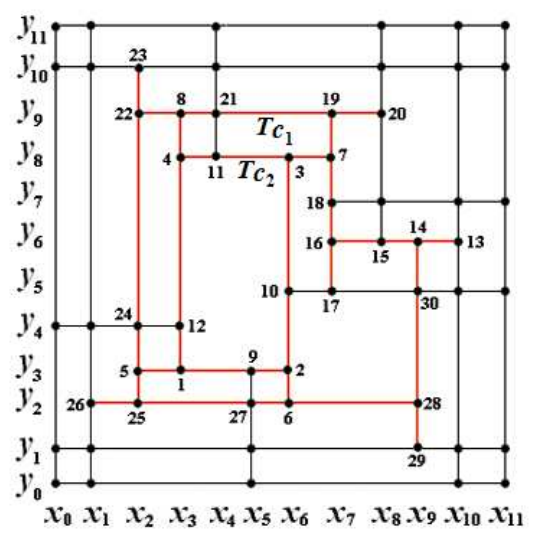

Figure 12: A T-mesh with a 2-nested mixed T-cycle $\mathscr{T}_{2}^{\prime}$.

The two singularity factors $a_{1}$ and $a_{2}$ corresponding to $T c_{1}$ and $T c_{2}$ are

$$
\begin{aligned}
& a_{1}=\frac{s_{7,10}}{s_{4,7}} \frac{s_{2,8} s_{2,4}}{s_{9,10} s_{8,9}} \frac{s_{5,9} s_{1,9}}{s_{2,5} s_{1,2}} \frac{t_{1,6}}{t_{6,7}} \frac{t_{7,9} t_{5,9}}{t_{2,5} t_{1,2}} \frac{t_{2,10} t_{2,4}}{t_{4,9} t_{9,10}}, \\
& a_{2}=\frac{s_{5,6} s_{2,6}}{s_{3,5} s_{2,3}} \frac{s_{3,7} s_{3,4}}{s_{4,6} s_{6,7}} \frac{t_{5,8} t_{2,8}}{t_{3,5} t_{2,3}} \frac{t_{3,9} t_{3,4}}{t_{4,8} t_{8,9}} .
\end{aligned}
$$

Theorem 3.5. The dimension of spline space $S\left(2,2,1,1, \mathscr{T}_{2}^{\prime}\right)$ over $T$-mesh $\mathscr{T}_{2}^{\prime}$ shown in Fig. 12 is unstable. The T-mesh's degeneration of two different cases are shown in Fig. 13.

$$
\begin{aligned}
& \operatorname{dim} S\left(2,2,1,1, \mathscr{T}_{2}^{\prime}\right) \\
= & \begin{cases}32, & \text { if } a_{1} \neq 1 \text { and } a_{2} \neq 1\left(\text { Both } T c_{1} \text { and } T c_{2} \text { are degenerated from } \mathscr{T}_{2}^{\prime}\right), \\
33, & \text { if } a_{1}=1 \text { and for any } a_{2}\left(T c_{2} \text { is degenerated from } \mathscr{T}_{2}^{\prime}\right), \\
33, & \text { if } a_{1} \neq 1 \text { and } a_{2}=1 \text { (None degeneration), }\end{cases}
\end{aligned}
$$

where $a_{1}$ and $a_{2}$ are defined by (3.2a) and (3.2b).

Here we omit this proof since it is similar to the proof of Theorem 3.4.

Fig. 13 shows two different cases on T-mesh's degeneration and we give the following examples in different cases.

Remark 3.2. (i) We set $\left(x_{0}, x_{1}, \cdots, x_{11}\right)=\left(-1,0,3, \frac{51}{12}, \frac{13}{3}, \frac{20}{3}, \frac{41}{6}, 7, \frac{22}{3}, 8, \frac{29}{3}, 10\right)$ and $\left(y_{0}, y_{1}, \cdots, y_{11}\right)=\left(-1,0, \frac{5}{9}, \frac{14}{9}, \frac{17}{9}, \frac{23}{9}, \frac{25}{9}, \frac{50}{9}, \frac{51}{9}, \frac{53}{9}, \frac{77}{9}, 9\right)$ in Fig. 12. By computing, $a_{1} \neq 1$ and $a_{2} \neq 1$, hence $\operatorname{dim} S\left(2,2,1,1, \mathscr{T}_{2}^{\prime}\right)=32$ and two $T$-cycles are totally degenerated from $\mathfrak{T}_{2}^{\prime}$.

(ii) We set $\left(x_{0}, x_{1}, \cdots, x_{11}\right)=\left(-1,0,4, \frac{51}{12}, \frac{13}{3}, \frac{20}{3}, \frac{41}{6}, 7, \frac{22}{3}, 8, \frac{29}{3}, 10\right)$ and $\left(y_{0}, y_{1}, \cdots\right.$, $\left.y_{11}\right)=\left(-1,0, \frac{5}{9}, \frac{14}{9}, \frac{17}{9}, \frac{23}{9}, \frac{25}{9}, \frac{50}{9}, \frac{51}{9}, \frac{53}{9}, \frac{77}{9}, 9\right)$ in Fig. 12. By computing, $a_{1}=1$ and $a_{2} \neq 1$, hence $\operatorname{dim} S\left(2,2,1,1, \mathscr{T}_{2}^{\prime}\right)=33, T c_{2}$ is degenerated from $\mathscr{T}_{2}^{\prime}$. 


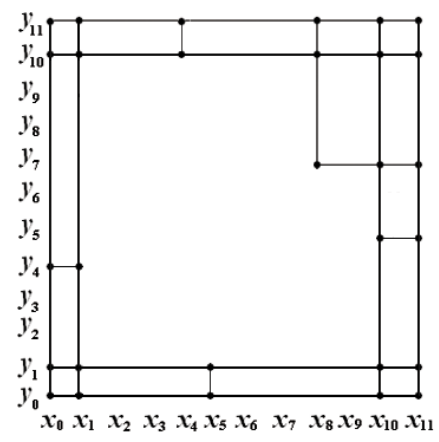

(a) Both of the two $T$-cycles are degenerated.

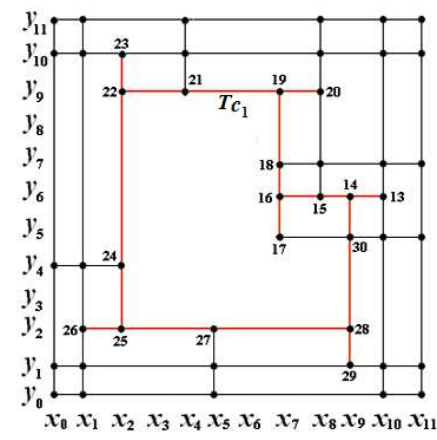

(b) The inner $T$-cycle $T c_{2}$ is degenerated.

Figure 13: The two degenerations of $T$-mesh $\mathscr{T}_{2}^{\prime}$.

(iii) We set $\left(x_{0}, x_{1}, \cdots, x_{11}\right)=\left(-1,0,4, \frac{43}{10}, \frac{13}{3}, \frac{20}{3}, \frac{34}{5}, 7, \frac{22}{3}, 8, \frac{29}{3}, 10\right)$ and $\left(y_{0}, y_{1}, \ldots\right.$, $\left.y_{11}\right)=\left(-1,0, \frac{5}{9}, \frac{174433}{59040}-\frac{1}{6560} \sqrt{64183089}, \frac{17}{9}, \frac{23}{9}, \frac{25}{9}, \frac{50}{9}, \frac{253}{45}, \frac{53}{9}, \frac{77}{9}, 9\right)$ in Fig. 12. By computing, $a_{1}=1$ and $a_{2}=1$, hence $\operatorname{dim} S\left(2,2,1,1, \mathscr{T}_{2}^{\prime}\right)=33, T c_{2}$ is degenerated from $\mathscr{T}_{2}^{\prime}$.

(iv) We set $\left(x_{0}, x_{1}, \cdots, x_{11}\right)=\left(-1,0,1,2, \frac{7}{3}, \frac{8}{3}, 3,4,5,6,7,8\right)$ and $\left(y_{0}, y_{1}, \cdots, y_{11}\right)$ $=\left(-1,0,1,2, \frac{7}{3}, \frac{8}{3}, \frac{59}{21}, \frac{62}{21}, 3,4,5,6\right)$ in Fig. 12. By computing, $a_{1} \neq 1$ and $a_{2}=1$, hence $\operatorname{dim} S\left(2,2,1,1, \mathscr{T}_{2}^{\prime}\right)=33$ and $\mathscr{T}_{2}^{\prime}$ is non-degenerate.

Comparing the structure of $\mathscr{T}_{2}^{\prime}$ with that of $\mathscr{T}_{2}$, similar results on the instability and structure's degeneration are derived. However, for other cases of more general nested Tcycles, such perfect results does not usually available due to its complexity of the structure. In fact, it is hard to discuss the dimension of spline spaces over some more general T-meshes with complex structure.

\subsection{The dimension of the spline space on a T-mesh with a 3-nested T-cycle}

Example 3.3. Fig. 14 shows a $T$-mesh with a 3 -nested $T$-cycle $\mathscr{T}_{3}$, which is directly added a new $T$-cycle outside of the above 2 -nested $T$-cycle in $\mathscr{T}_{2}$. In this case, we rename the three $T$-cycles:

$$
\begin{aligned}
& T c_{1}=\left\{e_{9}=\overline{v_{29} v_{26}}, \quad e_{10}=\overline{v_{30} v_{27}}, \quad e_{11}=\overline{v_{31} v_{28}}, e_{12}=\overline{v_{32} v_{25}}\right\} \text {, }
\end{aligned}
$$

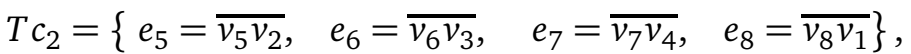

$$
\begin{aligned}
& T c_{3}=\left\{e_{1}=\overline{v_{12} v_{14}}, \quad e_{2}=\overline{v_{9} v_{15}}, \quad e_{3}=\overline{v_{10} v_{16}}, \quad e_{4}=\overline{v_{11} v_{13}}\right\} \text {. }
\end{aligned}
$$

The three singularity factors $a_{1}, a_{2}$ and $a_{3}$ corresponding to $T c_{1}, T c_{2}$ and $T c_{3}$ are 


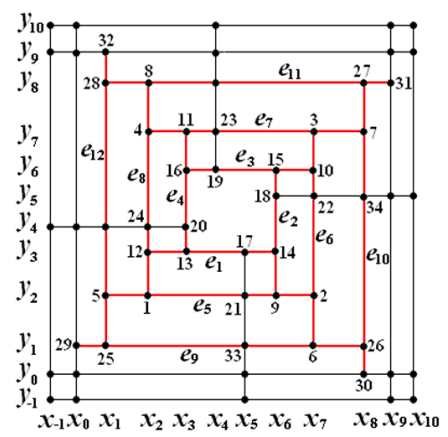

Figure 14: A T-mesh with a 3-nested T-cycle $\mathscr{T}_{3}$.

$$
\begin{aligned}
& a_{1}=\frac{s_{5,8} s_{0,8}}{s_{1,5} s_{0,1}} \frac{s_{1,9} s_{1,4}}{s_{4,8} s_{8,9}} \frac{t_{5,8} t_{0,8}}{t_{1,5} t_{0,1}} \frac{t_{1,9} t_{1,4}}{t_{4,8} t_{8,9}}, \\
& a_{2}=\frac{s_{5,7} s_{1,7}}{s_{1,2} s_{2,5}} \frac{s_{2,8} s_{2,4}}{s_{7,8} s_{4,7}} \frac{t_{5,7} t_{1,7}}{t_{1,2} t_{2,5}} \frac{t_{2,8} t_{2,4}}{t_{7,8} t_{4,7}}, \\
& a_{3}=\frac{s_{5,6} s_{2,6}}{s_{2,3} s_{3,5}} \frac{s_{3,7} s_{3,4}}{s_{6,7} s_{4,6}} \frac{t_{5,6} t_{2,6}}{t_{2,3} t_{3,5}} \frac{t_{3,7} t_{3,4}}{t_{6,7} t_{4,6}} .
\end{aligned}
$$

Conjecture 3.1. The dimension of spline space $S\left(2,2,1,1, \mathscr{T}_{3}\right)$ over $T$-mesh $\mathscr{T}_{3}$ shown in Fig. 14 is unstable. The T-mesh's degeneration in three different cases are showed in Fig. 15.

$\operatorname{dimS}\left(2,2,1,1, \mathscr{T}_{3}\right)$

$$
=\left\{\begin{array}{c}
29, \quad \begin{array}{c}
\text { if } a_{1} \neq 1, a_{2} \neq 1 \text { and } a_{3} \neq 1 \text { (All of } T c_{1}, T c_{2} \text { and } T c_{3} \text { are degenerated } \\
\text { from } \mathscr{T}_{3} \text { ), } \\
30, \quad \text { if } a_{1}=1 \text { and for any } a_{2} \text { and } a_{3} \text { (Both } T c_{2} \text { and } T c_{3} \text { are degenerated } \\
\quad \text { from } \mathscr{T}_{3} \text { ), }
\end{array} \\
30, \quad \text { if } a_{1} \neq 1, a_{2}=1 \text { and for any } a_{3}\left(T c_{3} \text { is degenerated from } \mathscr{T}_{3}\right. \text { ), } \\
30, \quad \text { if } a_{1} \neq 1, a_{2} \neq 1 \text { and } a_{3}=1 \text { (None degeneration), }
\end{array}\right.
$$

where $a_{1}, a_{2}$ and $a_{3}$ are defined by (3.3a), (3.3b) and (3.3c).

Analysis. See Appendix B.

We give the following examples in different cases of the $T$-mesh with a 3-nested $T$ cycle.

Remark 3.3. (i) We set $\left(x_{-1}, x_{0}, \cdots, x_{10}\right)=\left(-13,-12,-11, \frac{1}{2}, \frac{3}{2}, \frac{7}{3}, \frac{8}{3}, 3,4,16,17\right.$, 18) and $\left(y_{-1}, y_{0}, \cdots, y_{10}\right)=\left(-13,-12,-11, \frac{1}{2}, \frac{3}{2}, \frac{7}{3}, \frac{8}{3}, 3,4,16,17,18\right)$ in Fig. 14. By computing, $a_{1} \neq 1, a_{2} \neq 1$ and $a_{3} \neq 1, b_{1}=35401$, hence $\operatorname{dim} S(2,2,1,1$, $\left.\mathscr{T}_{3}\right)=29$ and three $T$-cycles are totally degenerated from $\mathscr{T}_{3}$.

(ii) We set $\left(x_{-1}, x_{0}, \cdots, x_{10}\right)=\left(-1092,-1091,-11, \frac{1}{2}, \frac{3}{2}, \frac{7}{3}, \frac{8}{3}, 3,4,16,1096,1097\right)$ and $\left(y_{-1}, y_{0}, \cdots, y_{10}\right)=\left(-1092,-1091,-11, \frac{1}{2}, \frac{3}{2}, \frac{7}{3}, \frac{8}{3}, 3,4,16,1096,1097\right)$ in Fig. 14. By computing, $a_{1}=1, a_{2} \neq 1$ and $a_{3} \neq 1, b_{1}=0.4016$, hence $\operatorname{dim} S(2,2,1,1$, $\left.\mathscr{T}_{3}\right)=30, T c_{2}$ and $T c_{3}$ are degenerated from $\mathscr{T}_{3}$. 


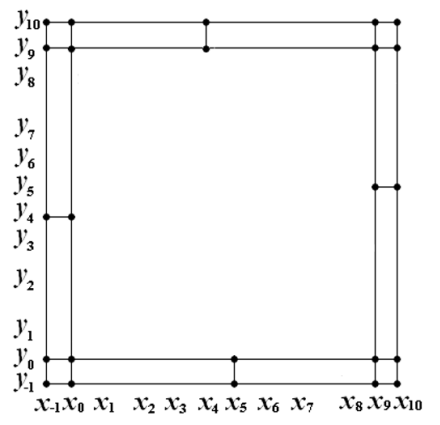

(a) All of the $T$-cycles are degenerated.

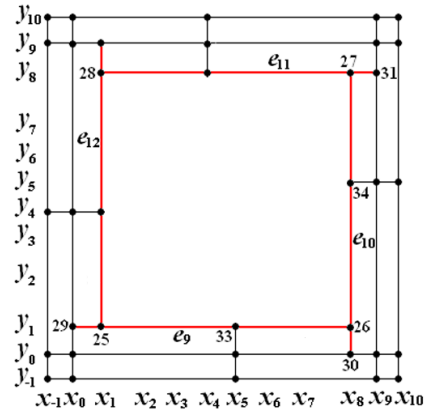

(b) The inner two $T$-cycles $T c_{2}$ and $T c_{3}$ are degenerated.

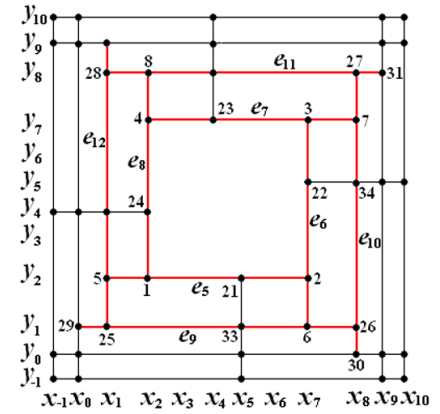

(c) The innermost $T$-cycle $T c_{3}$ is degenerated.

Figure 15: The three degenerations of $T$-mesh $\mathscr{T}_{3}$.

(iii) We set $\left(x_{-1}, x_{0}, \cdots, x_{10}\right)=\left(-1092,-1091,-11,1, \frac{3}{2}, \frac{7}{3}, \frac{8}{3}, 3,4,16,1096,1097\right)$ and $\left(y_{-1}, y_{0}, \cdots, y_{10}\right)=\left(-1092,-1091,-11,1, \frac{3}{2}, \frac{7}{3}, \frac{8}{3}, 3,4,16,1096,1097\right)$ in Fig. 14. By computing, $a_{1}=1, a_{2}=1$ and $a_{3} \neq 1, b_{1}=0.3957$, hence $\operatorname{dim} S(2,2,1,1$, $\left.\mathscr{T}_{3}\right)=30, T c_{2}$ and $T c_{3}$ are degenerated from $\mathscr{T}_{3}$.

(iv) We set $\left(x_{-1}, x_{0}, \cdots, x_{10}\right)=\left(-1092,-1091,-11,1,2, \frac{7}{3}, \frac{8}{3}, 3,4,16,1096,1097\right)$ and $\left(y_{-1}, y_{0}, \cdots, y_{10}\right)=\left(-1092,-1091,-11,1,2, \frac{7}{3}, \frac{8}{3}, 3,4,16,1096,1097\right)$ in Fig. 14. By computing, $a_{1}=1, a_{2}=1$ and $a_{3}=1, b_{1}=0.3957$, hence $\operatorname{dim} S(2,2,1,1$, $\left.\mathscr{T}_{3}\right)=30, T c_{2}$ and $T c_{3}$ are degenerated from $\mathscr{T}_{3}$.

(v) We set $\left(x_{-1}, x_{0}, \cdots, x_{10}\right)=\left(-36,-35,0,1,2, \frac{7}{3}, \frac{8}{3}, 3,4,5,40,41\right)$ and $\left(y_{-1}, y_{0}\right.$, $\left.\cdots, y_{10}\right)=\left(-36,-35,0,1,2, \frac{7}{3}, \frac{8}{3}, 3,4,5,40,41\right)$ in Fig. 14. By computing, $a_{1}=$ $1, a_{2} \neq 1$ and $a_{3}=1, b_{1}=1.8455$, hence $\operatorname{dim} S\left(2,2,1,1, \mathscr{T}_{3}\right)=30, T c_{2}$ and $T c_{3}$ are degenerated from $\mathscr{T}_{3}$.

(vi) We set $\left\{x_{-1}, x_{0}, \ldots, x_{10}\right)=\left(-13,-12,-11,1, \frac{3}{2}, \frac{7}{3}, \frac{8}{3}, 3,4,16,17,18\right)$ and $\left(y_{-1}\right.$, $\left.y_{0}, \ldots, y_{10}\right)=\left(-13,-12,-11,1, \frac{3}{2}, \frac{7}{3}, \frac{8}{3}, 3,4,16,17,18\right)$ in Fig. 14. By computing, $a_{1} \neq 1, a_{2}=1$ and $a_{3} \neq 1, b_{1}=33029$, hence $\operatorname{dim} S\left(2,2,1,1, \mathscr{T}_{3}\right)=30$ and $T c_{3}$ is degenerated from $\mathscr{T}_{3}$.

(vii) We set $\left(x_{-1}, x_{0}, \cdots, x_{10}\right)=\left(-13,-12,-11,1,2, \frac{7}{3}, \frac{8}{3}, 3,4,16,17,18\right)$ and $\left(y_{-1}\right.$, $\left.y_{0}, \cdots, y_{10}\right)=\left(-13,-12,-11,1,2, \frac{7}{3}, \frac{8}{3}, 3,4,16,17,18\right)$ in Fig.14. By computing, $a_{1} \neq 1, a_{2}=1$ and $a_{3}=1, b_{1}=33029$, hence $\operatorname{dim} S\left(2,2,1,1, \mathscr{T}_{3}\right)=30, T c_{3}$ is degenerated from $\mathscr{T}_{3}$.

(viii) We set $\left(x_{-1}, x_{0}, \cdots, x_{10}\right)=\left(-1092,-1091,-10,1,2, \frac{7}{3}, \frac{8}{3}, 3,4,16,1096,1097\right)$ and $\left(y_{-1}, y_{0}, \cdots, y_{10}\right)=\left(-1092,-1091,-10,1,2, \frac{7}{3}, \frac{8}{3}, 3,4,16,1096,1097\right)$ in Fig. 14. By computing, $a_{1} \neq 1, a_{2} \neq 1$ and $a_{3}=1, b_{1}=0.4145$, hence $\operatorname{dim} S(2,2,1,1$, $\left.\mathscr{T}_{3}\right)=30$ and $\mathscr{T}_{3}$ is non-degenerate. 


\subsection{A sufficient condition for the stability of dimension over $T$-mesh with a $N$-nested $T$-cycle}

Further, for the case with a $N$-nested T-cycle, we need to modify a constraint on the $T$-mesh similar to that in [18], in order to obtain the stable dimension formula of $S\left(m, n, \alpha, \beta, \mathscr{T}_{N}\right)$ over $T$-mesh with a $N$-nested T-cycle.

For the case of the $T$-mesh $\mathscr{T}_{2}$ with a 2-nested $T$-cycle, as shown in Fig. 10, there are four $T$-segments included in the innermost T-cycle $T c_{2}$. For each $T$-segment, there are only one mono-vertex and one multi-vertex except two end vertices. As shown in Appendix A, there is a conformality condition along each $T$-segment of $T c_{2}$. It is equivalent to the equation of (A.2), which is determined by the singularity factor. That is the reason for the instability in the dimension of spline space. According to the analysis in $[9,18]$, if there is one more mono-vertex in at least one of the $T$-segment of the $T$-cycle, then there is one more free conformality factor in (A.2). It implies there is no singularity factor for the $T$-cycle, i.e., the dimension is stable. By this idea, we have the following results of the stable dimension.

Theorem 3.6. Given a T-mesh $\mathscr{T}_{N}$ with a $N$-nested $T$-cycle, which includes $C_{h}$ horizontal cross-cuts, $C_{v}$ vertical cross-cuts, $T_{h}$ horizontal $T$-segments, $T_{v}$ vertical $T$-segments, and $V$ interior vertices. The $j$-th $T$-segment $e_{j}$ contains $h_{j}^{(1)}$ mono-vertices and $h_{j}^{(2)}$ multi-vertices except two end vertices. Suppose $h_{j}^{(1)}+2 \geq M_{0}$ (where $M_{0}=\frac{m+1}{m-\alpha}$ ) if $e_{j}$ is a horizontal $T$ segment and $h_{j}^{(1)}+2 \geq N_{0}$ (where $N_{0}=\frac{n+1}{n-\beta}$ ) if $e_{j}$ is a vertical T-segment. For each simple $T$-cycle, if there is at least one of the horizontal T-segments satisfies $h_{j}^{(1)}+1 \geq M_{0}$ or one of the vertical T-segments satisfies $h_{j}^{(1)}+1 \geq N_{0}$. Then the dimension of the spline space $S(m, n, \alpha, \beta, \mathscr{T})$ is

$$
\begin{aligned}
\operatorname{dim} S(m, n, \alpha, \beta, \mathscr{T})=(m & +1)(n+1)+\left(C_{h}-T_{h}\right)(m+1)(n-\beta) \\
& +\left(C_{v}-T_{v}\right)(n+1)(m-\alpha)+V(m-\alpha)(n-\beta) .
\end{aligned}
$$

Proof. By the conditions, there is no singularity factor for each $T$-cycle, since for each simple $T$-cycle, there is at least one of the horizontal $T$-segments satisfies $h_{j}^{(1)}+1 \geq M_{0}$ or one of the vertical $T$-segments satisfies $h_{j}^{(1)}+1 \geq N_{0}$. Thus, all the $T_{k} T$-segments in $\mathscr{T}_{N}$ can be arranged in an order from inner to outer $T$-cycles of $\mathscr{T}_{N}$ showed in Fig. 16 as:

$$
e_{T_{k}} \rightarrow e_{T_{k}-1} \rightarrow \cdots \rightarrow e_{2} \rightarrow e_{1},
$$

then the rest proof is similar to that in [18]. Here we omit this proof.

\section{A case of T-mesh with a pair of parallel $T$-cycles}

In this section, we discuss a case of $T$-mesh $\mathscr{T}_{P}$ which contains a pair of parallel $T$ cycles as shown in Fig. 17(a). It can be found that the dimension of spline space is stable, but this may lead to structure's degeneration. 


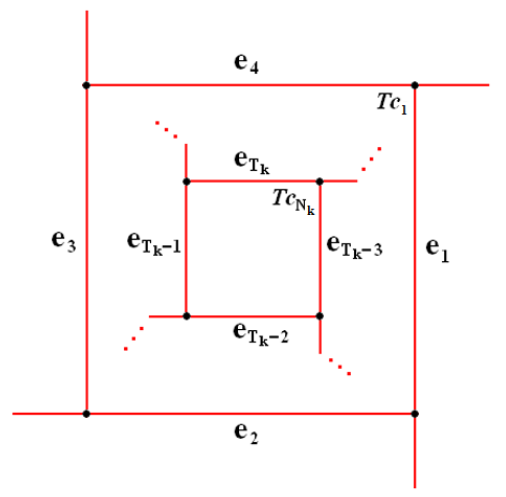

Figure 16: An order of $T$-segments in $\mathscr{T}_{N}$.

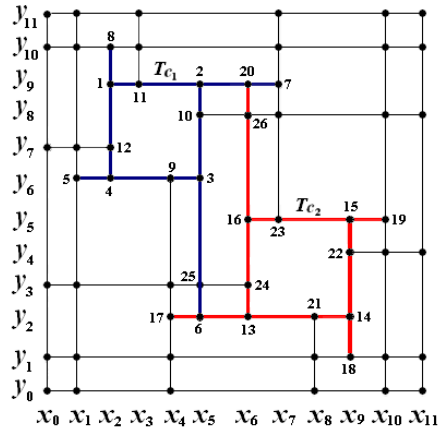

(a) A $T$-mesh with two parallel $T$ cycles $\mathscr{T}_{P}$

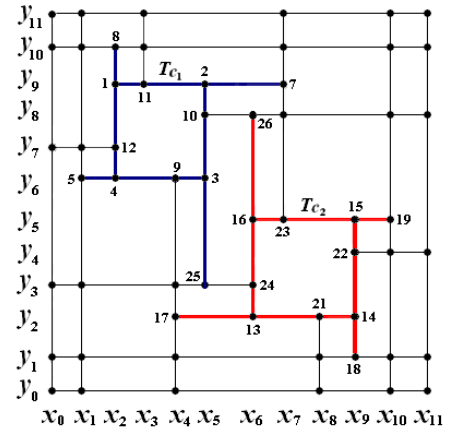

(b) $\mathscr{T}_{P}$ 's degeneration

Figure 17: A $T$-mesh with a pair of parallel $T$-cycles $\mathscr{T}_{P}$ and its degeneration.

Example 4.1. We consider the case of a given $T$-mesh with a pair of parallel $T$-cycles as shown in Fig. 17(a). Assume that $\mathbf{x}=\left(x_{i}\right)_{i=0}^{11}$ and $\mathbf{y}=\left(y_{i}\right)_{y=0}^{11}$ are two ascending sequences. There are two simple $T$-cycles

$$
T c_{1}=\left\{\overline{v_{5} v_{3}}, \overline{v_{6} v_{2}}, \overline{v_{7} v_{1}}, \overline{v_{8} v_{4}}\right\}, \quad T c_{2}=\left\{\overline{v_{17} v_{14}}, \overline{v_{18} v_{15}}, \overline{v_{19} v_{16}}, \overline{v_{20} v_{13}}\right\}
$$

The two singularity factors $a_{1}$ and $a_{2}$ corresponding to $T c_{1}$ and $T c_{2}$ are

$$
\begin{aligned}
& a_{1}=\frac{t_{6,8} t_{3,6}}{t_{3,9} t_{8,9}} \frac{s_{2,4} s_{1,2}}{s_{4,5} s_{1,5}} \frac{t_{9,10} t_{7,9}}{t_{6,7} t_{6,10}} \frac{s_{5,7} s_{3,5}}{s_{2,3} s_{2,7}}, \\
& a_{2}=\frac{t_{5,8} t_{3,5}}{t_{2,8} t_{2,3}} \frac{s_{9,10} s_{7,9}}{s_{6,7} s_{6,10}} \frac{t_{2,4} t_{1,2}}{t_{4,5} t_{1,5}} \frac{s_{6,8} s_{4,6}}{s_{8,9} s_{4,9}} .
\end{aligned}
$$

Proposition 4.1. We consider the spline space $S\left(2,2,1,1, \mathscr{T}_{P}\right)$ over the T-mesh $\mathscr{T}_{P}$ with a pair of parallel T-cycles as shown in Fig. 17(a). If the conditions of $a_{1}=1$ and $a_{2}=1$ are satisfied, where $a_{1}$ and $a_{2}$ are defined by (4.1a) and (4.1b), the structure of $\mathscr{T}_{P}$ will be separated into 
two independent T-cycles as shown in Fig. 17(b); Otherwise, it will not be degenerated. For the both cases, the dimension of $S\left(2,2,1,1, \mathscr{T}_{P}\right)$ is 37 unchanged.

Proof. See Appendix C.

\section{Conclusions}

In this paper, we introduce a class of $T$-meshes with the structure of $N$-nested $T$-cycles. Using the smoothing cofactor-conformality method, a constraint on $T$-mesh is introduced in order to obtain a stable dimension formula of spline space over $T$-mesh with a $N$-nested $T$-cycle. Moreover, we discuss the instability in the dimensions of spline spaces over $T$ meshes with 2-nested and 3-nested $T$-cycles. At last, we indicate that a pair of parallel $T$-cycles in $T$-mesh may lead to structure's degeneration. In the future, we will discuss the dimension of spline space and structure's degeneration for more general $T$-meshes.

\section{Appendix A: Proof of Theorem 3.4}

Proof. By using the smoothing cofactor-conformality method, the conformality conditions of the smoothing cofactors on each $T$-segment can be transformed into two kinds of conformality conditions of the conformality factors at each interior vertex [9]. Denote $d_{i}$ by the conformality cofactor at the interior vertex $v_{i}(i=1, \cdots, 24)$. For $T c_{2}$, there is a conformality condition along each $T$-segment:

$$
\begin{aligned}
d_{12}\left(x-x_{2}\right)^{2}+d_{13}\left(x-x_{3}\right)^{2}+d_{17}\left(x-x_{5}\right)^{2}+d_{14}\left(x-x_{6}\right)^{2} & \equiv 0, \\
d_{9}\left(y-y_{2}\right)^{2}+d_{14}\left(y-y_{3}\right)^{2}+d_{18}\left(y-y_{5}\right)^{2}+d_{15}\left(y-y_{6}\right)^{2} & \equiv 0, \\
d_{10}\left(x-x_{7}\right)^{2}+d_{15}\left(x-x_{6}\right)^{2}+d_{19}\left(x-x_{4}\right)^{2}+d_{16}\left(x-x_{3}\right)^{2} & \equiv 0, \\
d_{11}\left(y-y_{7}\right)^{2}+d_{16}\left(y-y_{6}\right)^{2}+d_{20}\left(y-y_{4}\right)^{2}+d_{13}\left(y-y_{3}\right)^{2} & \equiv 0 .
\end{aligned}
$$

Every conformality condition is equivalent to a linear system. For example, (A.1a) is $A d=$ 0 , where

$$
A=\left(\begin{array}{cccc}
1 & 1 & 1 & 1 \\
-2 x_{3} & -2 x_{6} & -2 x_{2} & -2 x_{5} \\
x_{3}^{2} & x_{6}^{2} & x_{2}^{2} & x_{5}^{2}
\end{array}\right) \quad \text { and } \quad d=\left(\begin{array}{c}
d_{13} \\
d_{14} \\
d_{12} \\
d_{17}
\end{array}\right) \text {. }
$$

Since $\operatorname{rank}(A)=3$, the dimension of the solution space of (A.1a) is 1 . That is, three of the conformality factors can be determined by the rest one, e.g., $d_{13}=\frac{s_{5,6} s_{2,6}}{s_{2,3} s_{3,5}} d_{14}$, where $s_{i, j}=x_{i}-x_{j}$.

Similarly, by solving the conformality conditions along $T$-segments $\mathrm{e}_{2}, \mathrm{e}_{3}$ and $\mathrm{e}_{4}$ of $T c_{2}$, we obtain

$$
d_{14}=\frac{t_{5,6} t_{2,6}}{t_{2,3} t_{3,5}} d_{15}, \quad d_{15}=\frac{s_{3,7} s_{3,4}}{s_{6,7} s_{4,6}} d_{16}, \quad d_{16}=\frac{t_{3,7} t_{3,4}}{t_{6,7} t_{4,6}} d_{13},
$$


where $t_{i, j}=y_{i}-y_{j}$. Then we have

$$
d_{13}=a_{2} d_{13},
$$

where $a_{2}=\frac{s_{5,6} s_{2,6}}{s_{2,3} s_{3,5}} \frac{t_{5,6} t_{2,6}}{t_{2,3} t_{3,5}} \frac{s_{3,7} s_{3,4}}{s_{6,7} s_{4,6}} \frac{t_{3,7} t_{3,4}}{t_{6,7} t_{4,6}}$, and $a_{2}$ is the singularity factor of $T c_{2}$. It is equivalent to

$$
\left(a_{2}-1\right) d_{13}=0 .
$$

For $T c_{1}$, there are another four conformality conditions along $T$-segments $e_{5}, e_{6}, e_{7}$ and $e_{8}$ :

$$
\begin{aligned}
& d_{5}\left(x-x_{1}\right)^{2}+d_{1}\left(x-x_{2}\right)^{2}+d_{21}\left(x-x_{5}\right)^{2}+d_{9}\left(x-x_{6}\right)^{2}+d_{2}\left(x-x_{7}\right)^{2} \equiv 0, \\
& d_{6}\left(y-y_{1}\right)^{2}+d_{2}\left(y-y_{2}\right)^{2}+d_{22}\left(y-y_{5}\right)^{2}+d_{10}\left(y-y_{6}\right)^{2}+d_{3}\left(y-y_{7}\right)^{2} \equiv 0, \\
& d_{7}\left(x-x_{8}\right)^{2}+d_{3}\left(x-x_{7}\right)^{2}+d_{23}\left(x-x_{4}\right)^{2}+d_{11}\left(x-x_{3}\right)^{2}+d_{4}\left(x-x_{2}\right)^{2} \equiv 0, \\
& d_{8}\left(y-y_{8}\right)^{2}+d_{4}\left(y-y_{7}\right)^{2}+d_{24}\left(y-y_{4}\right)^{2}+d_{12}\left(y-y_{3}\right)^{2}+d_{1}\left(y-y_{2}\right)^{2} \equiv 0 .
\end{aligned}
$$

Similarly, they are also equivalent to corresponding linear systems with the solution space of dimension 2 . We obtain

$$
\begin{array}{ll}
d_{1}=\frac{s_{5,7} s_{1,7}}{s_{1,2} s_{2,5}} d_{2}+\frac{s_{5,6} s_{1,6}}{s_{1,2} s_{2,5}} d_{9}, & d_{2}=\frac{t_{5,7} t_{1,7}}{t_{1,2} t_{2,5}} d_{3}+\frac{t_{5,6} t_{1,6}}{t_{1,2} t_{2,5}} d_{10}, \\
d_{3}=\frac{s_{2,8} s_{2,4}}{s_{7,8} s_{4,7}} d_{4}+\frac{s_{3,8} s_{3,4}}{s_{7,8} s_{4,7}} d_{11}, & d_{4}=\frac{t_{2,8} t_{2,4}}{t_{7,8} t_{4,7}} d_{1}+\frac{t_{3,8} t_{3,4}}{t_{7,8} t_{4,7}} d_{12} .
\end{array}
$$

Denote the coefficients by

$$
\begin{array}{llll}
c_{1,2}=\frac{s_{5,7} s_{1,7}}{s_{1,2} s_{2,5}}, & c_{1,9}=\frac{s_{5,6} s_{1,6}}{s_{1,2} s_{2,5}}, & c_{2,3}=\frac{t_{5,7} t_{1,7}}{t_{1,2} t_{2,5}}, & c_{2,10}=\frac{t_{5,6} t_{1,6}}{t_{1,2} t_{2,5}}, \\
c_{3,4}=\frac{s_{2,8} s_{2,4}}{s_{7,8} s_{4,7}}, & c_{3,11}=\frac{s_{3,8} s_{3,4}}{s_{7,8} s_{4,7}}, & c_{4,1}=\frac{t_{2,8} t_{2,4}}{t_{7,8} t_{4,7}}, & c_{4,12}=\frac{t_{3,8} t_{3,4}}{t_{7,8} t_{4,7}} .
\end{array}
$$

We have the following relation equation

$$
\begin{aligned}
d_{1} & =c_{1,2} d_{2}+c_{1,9} d_{9} \\
& =c_{1,2}\left(c_{2,3} d_{3}+c_{2,10} d_{10}\right)+c_{1,9} d_{9} \\
& =c_{1,2} c_{2,3}\left(c_{3,4} d_{4}+c_{3,11} d_{11}\right)+c_{1,2} c_{2,10} d_{10}+c_{1,9} d_{9} \\
& =c_{1,2} c_{2,3} c_{3,4}\left(c_{4,1} d_{1}+c_{4,12} d_{12}\right)+c_{1,2} c_{2,3} c_{3,11} d_{11}+c_{1,2} c_{2,10} d_{10}+c_{1,9} d_{9} \\
& =c_{1,2} c_{2,3} c_{3,4} c_{4,1} d_{1}+c_{1,2} c_{2,3} c_{3,4} c_{4,12} d_{12}+c_{1,2} c_{2,3} c_{3,11} d_{11}+c_{1,2} c_{2,10} d_{10}+c_{1,9} d_{9} .
\end{aligned}
$$

According to the conformality conditions of the $T$-segments of $T c_{2}$, we also have

$$
d_{12}=\frac{s_{5,6} s_{3,6}}{s_{2,5} s_{2,3}} \frac{t_{2,6} t_{2,5}}{t_{3,6} t_{3,5}} d_{9}, \quad d_{11}=\frac{t_{4,6}}{t_{5,6}} \frac{t_{2,5} t_{2,3}}{t_{3,7} t_{4,7}} \frac{s_{6,7} s_{4,6}}{s_{3,7} s_{3,4}} d_{9}, \quad d_{10}=\frac{s_{4,6} s_{3,6}}{s_{3,7} s_{4,7}} \frac{t_{2,5} t_{2,3}}{t_{5,6} t_{3,6}} d_{9} .
$$

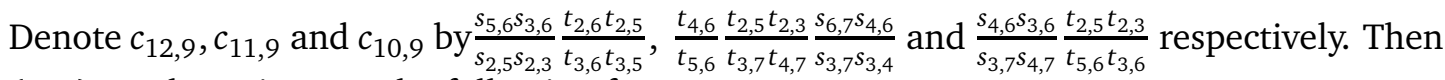
(A.4) can be written as the following form

$$
\left(a_{1}-1\right) d_{1}+b_{1} d_{9}=0,
$$


where $a_{1}=c_{1,2} c_{2,3} c_{3,4} c_{4,1}, a_{1}$ is the singularity factor of $T c_{1}$ and

$$
b_{1}=c_{1,2} c_{2,3} c_{3,4} c_{4,12} c_{12,9}+c_{1,2} c_{2,3} c_{3,11} c_{11,9}+c_{1,2} c_{2,10} c_{10,9}+c_{1,9}
$$

By the smoothing cofactor-conformality method, the dimension of the spline space $S(m, n, \alpha$, $\beta, \mathscr{T}_{2}$ ) is determined by

$$
\begin{aligned}
& \operatorname{dim} S\left(m, n, \alpha, \beta, \mathscr{T}_{2}\right) \\
= & (m+1)(n+1)+C_{h}(m+1)(n-\beta)+C_{v}(m-\alpha)(n+1)+\sigma,
\end{aligned}
$$

where $C_{h}$ and $C_{v}$ are the number of horizontal cross-cuts and vertical cross cuts in $\mathscr{T}_{2} . \sigma$ is the dimension of the solve space of the global conformality conditions of the 2-nested $T$-cycle, i.e., the conditions of (A.1a)-(A.1d), (A.3a)-(A.3d). Hence, the values of $a_{1}, a_{2}$ and $b_{1}$ in (A.2) and (A.6) determine the dimension of the spline space $S\left(2,2,1,1, \mathscr{T}_{2}\right)$.

We can easily see that $c_{i, j}$ metioned above are totally positive due to the ascending of $\mathbf{x}$ and $\mathbf{y}$ in $\mathscr{T}_{2}$. Hence, the $b_{1}$ determined by the expression of (A.7) has the property that $b_{1}>0$ for the $T$-mesh $\mathscr{T}_{2}$.

According to the values of $a_{1}$ and $a_{2}$, the dimension of the $T$-connected component with a 2-nested $T$-cycle can be briefly analyzed in the following cases.

Case 1: $a_{2} \neq 1$. (By (A.2), $d_{13}=0$, i.e. all of the conformality factors for $T c_{2}$ are zeros, then the inner $T$-cycle is degenerated. Therefore, $\left(a_{1}-1\right) d_{1}=0$.)

a) If $a_{1}=1$, then $d_{1}$ is a free variable and the dimension of $T$-connected component with a 2 -nested $T$-cycle is 1 .

b) If $a_{1} \neq 1$, then we have $d_{1}=0$, that is, the $T$-cycle $T c_{1}$ is also degenerated. The dimension of $T$-connected component with a 2-nested $T$-cycle is 0 .

Case 2: $a_{2}=1$.

a) If $a_{1}=1$, it follows that $d_{9}=0$ and $d_{1}$ is a free variable by (A.6). Then by (A.5), the inner $T$-cycle $T c_{2}$ is degenerated and the analysis of relevant dimension is the same as the case $1(\mathrm{a})$.

b) If $a_{1} \neq 1, d_{1}$ is determined by $d_{9}$ by (A.6), then determined by $d_{13}$ in (A.2) by the conformality conditions of the $T$-segments of $T c_{2}$. Since $a_{2}=1, d_{13}$ can not be zero. The dimension of $T$-connected component with a 2-nested $T$-cycle is 1 .

Therefore, if there is at least one condition of $a_{1}=1$ and $a_{2}=1$ satisfied, then the dimension of $S\left(2,2,1,1, \mathscr{T}_{2}\right)$ is 30 ; otherwise, the dimension is 29 . Then the proof is completed. 


\section{Appendix B: Analysis of conjecture 3.1}

Analysis. Assume that $\mathbf{x}=\left(x_{i}\right)_{i=-1}^{10}$ and $\mathbf{y}=\left(y_{i}\right)_{y=-1}^{10}$ are two ascending sequences. The conformality conditions of the $T$-segments of $T c_{2}$ and $T c_{3}$ are the same as the proof of Theorem 3.4. In addition, the conformality conditions along the $T$-segments $\mathrm{e}_{9}, \mathrm{e}_{10}, \mathrm{e}_{11}, \mathrm{e}_{12}$ must be taken into consideration for the calculation of $\operatorname{dim} S\left(2,2,1,1, \mathscr{T}_{3}\right)$. Specifically, they are

$$
\begin{aligned}
& d_{29}\left(x-x_{0}\right)^{2}+d_{25}\left(x-x_{1}\right)^{2}+d_{33}\left(x-x_{5}\right)^{2}+d_{6}\left(x-x_{7}\right)^{2}+d_{26}\left(x-x_{8}\right)^{2} \equiv 0, \\
& d_{30}\left(y-y_{0}\right)^{2}+d_{26}\left(y-y_{1}\right)^{2}+d_{34}\left(y-y_{5}\right)^{2}+d_{7}\left(y-y_{7}\right)^{2}+d_{27}\left(y-y_{8}\right)^{2} \equiv 0, \\
& d_{31}\left(x-x_{9}\right)^{2}+d_{27}\left(x-x_{8}\right)^{2}+d_{35}\left(x-x_{4}\right)^{2}+d_{8}\left(x-x_{2}\right)^{2}+d_{28}\left(x-x_{1}\right)^{2} \equiv 0, \\
& d_{32}\left(y-y_{9}\right)^{2}+d_{28}\left(y-y_{8}\right)^{2}+d_{36}\left(y-y_{4}\right)^{2}+d_{5}\left(y-y_{2}\right)^{2}+d_{25}\left(y-y_{1}\right)^{2} \equiv 0 .
\end{aligned}
$$

By solving the conformality conditions of (B.1a)-(B.1d), we obtain

$$
\begin{array}{rlrl}
d_{25} & =\frac{s_{5,8} s_{0,8}}{s_{1,5} s_{0,1}} d_{26}+\frac{s_{5,7} s_{0,7}}{s_{1,5} s_{0,1}} d_{6}, & d_{26} & =\frac{t_{5,8} t_{0,8}}{t_{1,5} t_{0,1}} d_{27}+\frac{t_{5,7} t_{0,7}}{t_{1,5} t_{0,1}} d_{7}, \\
d_{27}=\frac{s_{1,9} s_{1,4}}{s_{4,8} s_{8,9}} d_{28}+\frac{s_{2,9} s_{2,4}}{s_{4,8} s_{8,9}} d_{8}, & d_{28}=\frac{t_{1,9} t_{1,4}}{t_{4,8} t_{8,9}} d_{25}+\frac{t_{2,9} t_{2,4}}{t_{4,8} t_{8,9}} d_{5} .
\end{array}
$$

Denote $c_{25,26}, c_{25,6}, c_{26,27}, c_{26,7}, c_{27,28}, c_{27,8}, c_{28,25}, c_{28,5}$ by the coefficients

$$
\frac{s_{5,8} s_{0,8}}{s_{1,5} s_{0,1}}, \frac{s_{5,7} s_{0,7}}{s_{1,5} s_{0,1}}, \frac{t_{5,8} t_{0,8}}{t_{1,5} t_{0,1}}, \frac{t_{5,7} t_{0,7}}{t_{1,5} t_{0,1}}, \frac{s_{1,9} s_{1,4}}{s_{4,8} s_{8,9}}, \frac{s_{2,9} s_{2,4}}{s_{4,8} s_{8,9}}, \frac{t_{1,9} t_{1,4}}{t_{4,8} t_{8,9}}, \frac{t_{2,9} t_{2,4}}{t_{4,8} t_{8,9}}
$$

in above expressions respectively. It can be found that the above $c_{i, j}>0$.

For $T c_{1}$, we have the following relation equation

$$
\begin{aligned}
d_{25}= & c_{25,26} d_{26}+c_{25,6} d_{6} \\
= & c_{25,26}\left(c_{26,27} d_{27}+c_{26,7} d_{7}\right)+c_{25,6} d_{6} \\
= & c_{25,26} c_{26,27}\left(c_{27,28} d_{28}+c_{27,8} d_{8}\right)+c_{25,26} c_{26,7} d_{7}+c_{25,6} d_{6} \\
= & c_{25,26} c_{26,27} c_{27,28}\left(c_{28,25} d_{25}+c_{28,5} d_{5}\right)+c_{25,26} c_{26,27} c_{27,8} d_{8}+c_{25,26} c_{26,7} d_{7}+c_{25,6} d_{6} \\
= & c_{25,26} c_{26,27} c_{27,28} c_{28,25} d_{25}+c_{25,26} c_{26,27} c_{27,28} c_{28,5} d_{5}+c_{25,26} c_{26,27} c_{27,8} d_{8} \\
& \quad+c_{25,26} c_{26,7} d_{7}+c_{25,6} d_{6} .
\end{aligned}
$$

The conformality factors of $d_{5}, d_{8}, d_{7}$ can be transformed into $d_{6}$ according to the conformality conditions of the $T$-segments of $T c_{2}$ and $T c_{3}$. For the three $T$-cycles $T c_{1}, T c_{2}$ and $T c_{3}$, we define the corresponding singularity factors $a_{1}, a_{2}$ and $a_{3}$ respectively:

$$
\begin{aligned}
& a_{1}=\frac{s_{5,8} s_{0,8}}{s_{1,5} s_{0,1}} \frac{s_{1,9} s_{1,4}}{s_{4,8} s_{8,9}} \frac{t_{5,8} t_{0,8}}{t_{1,5} t_{0,1}} \frac{t_{1,9} t_{1,4}}{t_{4,8} t_{8,9}}, \\
& a_{2}=\frac{s_{5,7} s_{1,7}}{s_{1,2} s_{2,5}} \frac{s_{2,8} s_{2,4}}{s_{7,8} s_{4,7}} \frac{t_{5,7} t_{1,7}}{t_{1,2} t_{2,5}} \frac{t_{2,8} t_{2,4}}{t_{7,8} t_{4,7}}, \\
& a_{3}=\frac{s_{5,6} s_{2,6}}{s_{2,3} s_{3,5}} \frac{s_{3,7} s_{3,4}}{s_{6,7} s_{4,6}} \frac{t_{5,6} t_{2,6}}{t_{2,3} t_{3,5}} \frac{t_{3,7} t_{3,4}}{t_{6,7} t_{4,6}} .
\end{aligned}
$$


It can be obtained that

$$
\begin{aligned}
& d_{5}=\frac{-\frac{s_{5,6} s_{2,6}}{s_{1,2} s_{1,5}}+\frac{s_{2,7} s_{2,5}}{s_{1,5} s_{1,7}}\left(\frac{b_{2}}{a_{2}-1}+\frac{s_{5,6} s_{1,6}}{s_{1,2} s_{2,5}}\right)}{\frac{t_{6,7} t_{2,5}}{t_{1,7} t_{1,5}} \frac{s_{4,6} s_{3,6}}{s_{3,7} s_{4,7}} \frac{t_{2,3}}{t_{3,6}}+\frac{t_{2,7} t_{2,5}}{t_{1,7} t_{1,5}} \frac{s_{1,2} s_{2,5}}{s_{5,7} s_{1,7}}\left(\frac{b_{2}}{a_{2}-1}+\frac{s_{5,6} s_{1,6}}{s_{1,2} s_{2,5}}\right)} d_{6}, \\
& d_{8}=\left(-\frac{b_{2}}{a_{2}-1}\right) \cdot \frac{-\frac{t_{2,7} t_{2,4}}{t_{7,8} t_{4,8}}+\frac{t_{3,7} t_{3,4}}{t_{7,8} t_{4,8}} \frac{t_{2,8} t_{2,4}}{t_{7,8} t_{4,7}} \frac{1}{\left(\frac{b_{2}}{a_{2}-1}+\frac{t_{3,8} t_{3,4}}{t_{7,8} t_{4,7}}\right)}}{\frac{t_{6,7} t_{2,5}}{t_{1,7} t_{1,5}} \frac{s_{4,6} s_{3,6}}{s_{3,7} s_{4,7}} \frac{t_{2,3}}{t_{3,6}}+\frac{t_{2,7} t_{2,5}}{t_{1,7} t_{1,5}} \frac{s_{1,2} s_{2,5}}{s_{5,7} s_{1,7}}\left(\frac{b_{2}}{a_{2}-1}+\frac{s_{5,6} s_{1,6}}{s_{1,2} s_{2,5}}\right)} d_{6}, \\
& d_{7}=\frac{\frac{s_{2,3} s_{6,7}}{s_{2,8} s_{4,8}} \frac{t_{4,6} t_{3,6}}{t_{3,7} t_{4,7}} \frac{s_{4,7}}{s_{3,6}}+\frac{s_{4,7} s_{2,7}}{s_{2,8} s_{4,8}} \frac{t_{1,2} t_{2,5}}{t_{5,7} t_{1,7}}\left(\frac{\overline{\bar{b}}_{2}}{a_{2}-1}+\frac{t_{5,6} t_{1,6}}{t_{1,2} t_{2,5}}\right)}{-\frac{t_{5,6} t_{2,6}}{t_{1,2} t_{1,5}}+\frac{t_{2,7} t_{2,5}}{t_{1,5} t_{1,7}}\left(\frac{\overline{\bar{b}}_{2}}{a_{2}-1}+\frac{t_{5,6} t_{1,6}}{t_{1,2} t_{2,5}}\right)} d_{6},
\end{aligned}
$$

where

$$
\begin{aligned}
b_{2}= & \frac{s_{5,7} s_{1,7}}{s_{1,2} s_{2,5}} \frac{t_{5,7} t_{1,7}}{t_{1,2} t_{2,5}} \frac{s_{2,8} s_{2,4}}{s_{7,8} s_{4,7}} \frac{t_{3,8}}{t_{7,8} t_{4,7}} \frac{t_{3,4}}{s_{2,5} s_{2,3}} \frac{s_{5,6} s_{3,6}}{t_{3,6} t_{3,5}}+\frac{t_{2,7} t_{2,5}}{s_{1,2} s_{2,5}} \frac{s_{5,7} s_{1,7}}{t_{1,2} t_{2,5}} \frac{t_{5,8} t_{1,7}}{s_{7,8} s_{4,7}} \frac{s_{3,5} s_{3,6}}{t_{5,6}} \\
& \frac{t_{2,5} t_{2,3}}{t_{3,7} t_{4,7}} \frac{s_{6,7} s_{4,6}}{s_{3,7} s_{3,4}}+\frac{s_{5,7} s_{1,7}}{s_{1,2} s_{2,5}} \frac{t_{5,6} t_{1,6}}{t_{1,2} t_{2,5}} \frac{s_{4,6} s_{3,6}}{s_{3,7} s_{4,7}} \frac{t_{2,5} t_{2,3}}{t_{5,6} t_{3,6}}+\frac{s_{5,6} s_{1,6}}{s_{1,2} s_{2,5}}, \\
\bar{b}_{2}= & \frac{t_{2,8} t_{2,4}}{t_{7,8} t_{4,7}} \frac{s_{5,7} s_{1,7}}{s_{1,2} s_{2,5}} \frac{t_{5,7} t_{1,7}}{t_{1,2}} \frac{t_{2,5}}{s_{3,8} s_{3,4}} \frac{t_{3,6} t_{3,4} s_{4,7}}{s_{4,7} t_{6,7}} \frac{s_{2,6} s_{2,5}}{s_{3,6} s_{3,5}}+\frac{t_{2,8} t_{2,4}}{t_{7,8} t_{4,7}} \frac{s_{5,7} s_{1,7}}{s_{1,2} s_{2,5}} \frac{t_{5,6} t_{1,6}}{t_{1,2} t_{2,5}} \frac{s_{4,6}}{s_{4,7}} \\
& \frac{t_{2,3} t_{3,5}}{t_{5,6} t_{2,6}} \frac{s_{2,5} s_{2,3}}{s_{5,6} s_{3,7}}+\frac{t_{2,8} t_{2,4}}{t_{7,8} t_{4,7}} \frac{s_{5,6} s_{1,6}}{s_{1,2} s_{2,5}} \frac{t_{3,6}}{t_{3,6}} \frac{t_{3,5}}{t_{2,5} s_{2,3}}+\frac{t_{3,8}}{s_{3,6} s_{5,6}}+\frac{t_{3,4}}{t_{7,8} t_{4,7}}, \\
\overline{\bar{b}}_{2}= & \frac{t_{5,7} t_{1,7}}{t_{1,2} t_{2,5}} \frac{s_{2,8} s_{2,4}}{s_{7,8} s_{4,7}} \frac{t_{2,8} t_{2,4}}{t_{7,8} t_{4,7}} \frac{s_{5,6} s_{1,6}}{s_{1,2} s_{2,5}} \frac{s_{3,7} s_{4,7}}{s_{4,6} s_{3,6}} \frac{t_{5,6} t_{3,6}}{t_{2,5} t_{2,3}}+\frac{t_{5,7} t_{1,7}}{t_{1,2} t_{2,5}} \frac{s_{2,8} s_{2,4}}{s_{7,8} s_{4,7}} \frac{t_{3,8} t_{3,4}}{t_{7,8} t_{4,7}} \frac{s_{5,6}}{s_{4,6}} \\
& \frac{s_{4,7} s_{3,7}}{s_{2,5} s_{2,3}} \frac{t_{5,6} t_{2,6}}{t_{2,3} t_{3,5}}+\frac{t_{5,7} t_{1,7}}{t_{1,2} t_{2,5}} \frac{s_{3,8} s_{3,4}}{s_{7,8} s_{4,7}} \frac{t_{4,6} t_{3,6}}{t_{3,7} t_{4,7}} \frac{s_{4,7} s_{6,7}}{s_{3,6} s_{3,4}}+\frac{t_{5,6} t_{1,6}}{t_{1,2} t_{2,5}} .
\end{aligned}
$$

The coefficients of $b_{2}, \bar{b}_{2}, \overline{\bar{b}}_{2}$ come from the following relation of the conformality factors.

$$
\begin{aligned}
& \left(a_{2}-1\right) d_{1}+b_{2} d_{9}=0 \\
& \left(a_{2}-1\right) d_{4}+\bar{b}_{2} d_{12}=0 \\
& \left(a_{2}-1\right) d_{2}+\overline{\bar{b}}_{2} d_{10}=0 .
\end{aligned}
$$

Denote $r_{5,6}, r_{8,6}$ and $r_{7,6}$ by the correlation coefficients of (B.3), i.e., $d_{5}=r_{5,6} d_{6}, d_{8}=r_{8,6} d_{6}$ and $d_{7}=r_{7,6} d_{6}$. Then the equation of (B.2) can be written as

$$
\left(a_{1}-1\right) d_{25}+b_{1} d_{6}=0,
$$

where $a_{1}=c_{25,26} c_{26,27} c_{27,28} c_{28,25}$ and

$$
b_{1}=c_{25,26} c_{26,27} c_{27,28} c_{28,5} r_{5,6}+c_{25,26} c_{26,27} c_{27,8} r_{8,6}+c_{25,26} c_{26,7} r_{7,6}+c_{25,6} \text {. }
$$


Consequently, the dimension of $T$-connected component of a 3-nested $T$-cycle can be decided by the following system:

$$
\begin{aligned}
& \left(a_{3}-1\right) d_{13}=0, \\
& \left(a_{2}-1\right) d_{1}+b_{2} d_{9}=0, \\
& \left(a_{1}-1\right) d_{25}+b_{1} d_{6}=0 .
\end{aligned}
$$

According to the analysis of Appendix A, we obtain $b_{2}>0$. Due to the complexity of the expressions of $r_{5,6}, r_{8,6}$ and $r_{7,6}$ in (B.3), here we make a conjecture that $b_{1}>0$ and we use a numerical method to test the conjecture in the following example.

Example B.1. We choose $x_{2}=1, x_{3}=2, x_{4}=\frac{7}{3}, x_{5}=\frac{8}{3}, x_{6}=3, x_{7}=4, y_{2}=1, y_{3}=$ $2, y_{4}=\frac{7}{3}, y_{5}=\frac{8}{3}, y_{6}=3, y_{7}=4$ in Fig. 14. By calculation, $a_{3}=0$. Then $r_{5,6}, r_{8,6}$ and $r_{7,6}$ are simplified into the following formulas.

$$
\begin{aligned}
& r_{5,6}=\frac{\left(-8+3 y_{1}\right)\left(4\left(-828+y_{1}\left(332-87 y_{8}\right)+223 y_{8}\right)+x_{8}\left(1212-367 y_{8}+y_{1}\left(-428+123 y_{8}\right)\right)\right)}{\left(-8+3 x_{1}\right)\left(-912+x_{8}\left(412-187 y_{8}\right)+312 y_{8}+x_{1}\left(848-248 y_{8}+3 x_{8}\left(-116+41 y_{8}\right)\right)\right)}, \\
& r_{8,6}=\frac{\left(-8+3 y_{1}\right)\left(-2748+x_{8}\left(943-367 y_{1}\right)+1212 y_{1}+x_{1}\left(812-428 y_{1}+3 x_{8}\left(-89+41 y_{1}\right)\right)\right)}{\left(-7+3 y_{8}\right)\left(-912+x_{8}\left(412-187 y_{8}\right)+312 y_{8}+x_{1}\left(848-248 y_{8}+3 x_{8}\left(-116+41 y_{8}\right)\right)\right)}, \\
& r_{7,6}=\frac{\left.\left(-8+3 y_{1}\right)\left(-648+y_{1}\left(392-267 y_{8}\right)+523 y_{8}\right)+x_{1}\left(312-187 y_{8}+y_{1}\left(-248+123 y_{8}\right)\right)\right)}{\left(-7+3 x_{8}\right)\left(-912+x_{8}\left(412-187 y_{8}\right)+312 y_{8}+x_{1}\left(848-248 y_{8}+3 x_{8}\left(-116+41 y_{8}\right)\right)\right)} .
\end{aligned}
$$

We use a numerical method to estimate the values of $r_{5,6}, r_{8,6}$ and $r_{7,6}$. Let $x_{1}, y_{1}$ be two real numbers of the range between -3 and $1, x_{8}, y_{8}$ be two real numbers of the range between 4 and 8 . We choose the step of 0.01 , using the Matlab software, it can be calculated that the values of $r_{5,6}, r_{8,6}$ and $r_{7,6}$ are greater than zero. Since all of $c_{i, j}>0$ in the expression of $b_{1}$ in (B.6), then it is easy to see $b_{1}>0$.

According to the values of $a_{1}, a_{2}$ and $a_{3}$, we omit the analysis of the dimension of spline space over $T$-mesh with a 3-nested $T$-cycle since it is similar to the proof of Theorem 3.4.

The analysis is completed.

\section{Appendix C: Proof of Proposition 4.1}

In order to prove Proposition 4.1, we firstly give the following lemma.

Lemma C.1. For $\mathscr{T}_{P}$ in Fig. 17(a), the relation of $\frac{s_{5,8} s_{4,5}}{s_{6,8} s_{4,6}}=\frac{s_{5,7} s_{3,5}}{s_{6,7} s_{3,6}}$ can not be satisfied.

Proof. Let $s_{3,5}=c, s_{6,7}=b, s_{4,5}=\widetilde{c}, s_{6,8}=\widetilde{b}, s_{5,6}=k$, where $c>\widetilde{c}>0, \widetilde{b}>b>0$ and $k>0$. We only need to testify the following equation can't be satisfied for $\mathscr{T}_{P}$. Suppose,

$$
\frac{(k+\widetilde{b}) \cdot \widetilde{c}}{\widetilde{b} \cdot(\widetilde{c}+k)}=\frac{(k+b) \cdot c}{b \cdot(c+k)} .
$$


Since the two sides of equation have the same form, we just need to consider $\frac{(k+b) \cdot c}{b \cdot(c+k)}=\lambda>$ 0 . If $\lambda$ and $k$ are regarded as two fixed values, then $b$ can be written by a function related to $c$.

$$
b(c)=\frac{k c}{(\lambda-1) c+\lambda k} .
$$

Take the derivative, we get

$$
b^{\prime}(c)=\frac{\lambda k^{2}}{[(\lambda-1) c+\lambda k]^{2}} .
$$

It is easy to find $b^{\prime}(c)>0$ under the assumption. It implies that $b$ increases with the increasing $c$, hence $c>\widetilde{c}$ gives $b>\widetilde{b}$. In other words, there won't be $c>\widetilde{c}$ and $\widetilde{b}>b$ such that satisfy the condition of

$$
\frac{(k+\tilde{b}) \cdot \widetilde{c}}{\widetilde{b} \cdot(\widetilde{c}+k)}=\frac{(k+b) \cdot c}{b \cdot(c+k)} .
$$

So the hypothesis is not correct.

Proof. Proof of Proposition 4.1. By solving the conformality conditions along $T$ segments of $T c_{1}$, we derive

$$
\begin{aligned}
d_{2} & =\frac{t_{6,8} t_{3,6}}{t_{3,9} t_{8,9}} d_{3}-\frac{t_{2,8} t_{2,3}}{t_{3,9} t_{8,9}} d_{6}, & d_{3} & =\frac{s_{2,4} s_{1,2}}{s_{4,5} s_{1,5}} d_{4}, \\
d_{4} & =\frac{t_{9,10} t_{7,9}}{t_{6,7} t_{6,10}} d_{1}, & d_{1} & =\frac{s_{5,7} s_{3,5}}{s_{2,3} s_{2,7}} d_{2}+\frac{s_{6,7} s_{3,6}}{s_{2,3} s_{2,7}} d_{20} .
\end{aligned}
$$

We have the following relation equation

$$
d_{2}=\frac{t_{6,8} t_{3,6}}{t_{3,9} t_{8,9}} \frac{s_{2,4} s_{1,2}}{s_{4,5} s_{1,5}} \frac{t_{9,10} t_{7,9}}{t_{6,7} t_{6,10}} \frac{s_{5,7} s_{3,5}}{s_{2,3} s_{2,7}} d_{2}+\frac{t_{6,8} t_{3,6}}{t_{3,9} t_{8,9}} \frac{s_{2,4} s_{1,2}}{s_{4,5} s_{1,5}} \frac{t_{9,10} t_{7,9}}{t_{6,7} t_{6,10}} \frac{s_{6,7} s_{3,6}}{s_{2,3} s_{2,7}} d_{20}-\frac{t_{2,8} t_{2,3}}{t_{3,9} t_{8,9}} d_{6} .
$$

It is equivalent to

$$
\left(1-a_{1}\right) d_{2}=\frac{t_{6,8} t_{3,6}}{t_{3,9} t_{8,9}} \frac{s_{2,4} s_{1,2}}{s_{4,5} s_{1,5}} \frac{t_{9,10} t_{7,9}}{t_{6,7} t_{6,10}} \frac{s_{6,7} s_{3,6}}{s_{2,3} s_{2,7}} d_{20}-\frac{t_{2,8} t_{2,3}}{t_{3,9} t_{8,9}} d_{6},
$$

where

$$
a_{1}=\frac{t_{6,8} t_{3,6}}{t_{3,9} t_{8,9}} \frac{s_{2,4} s_{1,2}}{s_{4,5} s_{1,5}} \frac{t_{9,10} t_{7,9}}{t_{6,7} t_{6,10}} \frac{s_{5,7} s_{3,5}}{s_{2,3} s_{2,7}} .
$$

By solving the conformality conditions along $T$-segments of $T c_{2}$, it is derived that

$$
\begin{aligned}
d_{13} & =\frac{t_{5,8} t_{3,5}}{t_{2,8} t_{2,3}} d_{16}-\frac{t_{8,9} t_{3,9}}{t_{2,8} t_{2,3}} d_{20}, & d_{15} & =\frac{t_{2,4} t_{1,2}}{t_{4,5} t_{1,5}} d_{14}, \\
d_{14} & =\frac{s_{6,8} s_{4,6}}{s_{8,9} s_{4,9}} d_{13}+\frac{s_{5,8} s_{4,5}}{s_{8,9} s_{4,9}} d_{6}, & d_{16} & =\frac{s_{9,10} s_{7,9}}{s_{6,7} s_{6,10}} d_{15} .
\end{aligned}
$$


We have the following relation equation

$$
\begin{aligned}
d_{13}= & \frac{t_{5,8} t_{3,5}}{t_{2,8} t_{2,3}} \frac{s_{9,10}}{s_{6,7} s_{6,10}} \frac{s_{7,9}}{t_{4,5} t_{1,5}} \frac{t_{2,8} t_{1,2}}{s_{8,9} s_{4,9}} d_{13} \\
& +\frac{t_{5,8} t_{3,5}}{t_{2,8} t_{2,3}} \frac{s_{9,10} s_{7,9}}{s_{6,7} s_{6,10}} \frac{t_{2,4} t_{1,2}}{t_{4,5} t_{1,5}} \frac{s_{5,8} s_{4,5}}{s_{8,9} s_{4,9}} d_{6}-\frac{t_{8,9} t_{3,9}}{t_{2,8} t_{2,3}} d_{20} .
\end{aligned}
$$

It follows that

$$
\left(1-a_{2}\right) d_{13}=\frac{t_{5,8} t_{3,5}}{t_{2,8} t_{2,3}} \frac{s_{9,10} s_{7,9}}{s_{6,7} s_{6,10}} \frac{t_{2,4} t_{1,2}}{t_{4,5} t_{1,5}} \frac{s_{5,8} s_{4,5}}{s_{8,9} s_{4,9}} d_{6}-\frac{t_{8,9} t_{3,9}}{t_{2,8} t_{2,3}} d_{20}
$$

where

$$
a_{2}=\frac{t_{5,8} t_{3,5}}{t_{2,8} t_{2,3}} \frac{s_{9,10} s_{7,9}}{s_{6,7} s_{6,10}} \frac{t_{2,4} t_{1,2}}{t_{4,5} t_{1,5}} \frac{s_{6,8} s_{4,6}}{s_{8,9} s_{4,9}} .
$$

Case 1: If $a_{1}=1$ and $a_{2}=1$, we have

$$
\begin{aligned}
& \frac{s_{6,7} s_{3,6}}{s_{5,7} s_{3,5}} d_{20}=\frac{t_{2,8} t_{2,3}}{t_{3,9} t_{8,9}} d_{6}, \\
& \frac{s_{5,8} s_{4,5}}{s_{6,8} s_{4,6}} d_{6}=\frac{t_{8,9} t_{3,9}}{t_{2,8} t_{2,3}} d_{20} .
\end{aligned}
$$

From (C.3a), substituting

into (C.3b)gives

$$
d_{20}=\frac{t_{2,8} t_{2,3}}{t_{3,9} t_{8,9}} \frac{s_{5,7} s_{3,5}}{s_{6,7} s_{3,6}} d_{6}
$$

$$
\left(\frac{s_{5,8} s_{4,5}}{s_{6,8} s_{4,6}}-\frac{s_{5,7} s_{3,5}}{s_{6,7} s_{3,6}}\right) d_{6}=0 .
$$

By Lemma C.1, we derive $d_{6}=0$ and $d_{20}=0$ under the conditions of $a_{1}=1$ and $a_{2}=1$. Thus the structure of the parallel $T$-cycles is separated into two independent $T$ cycles as shown in Fig. 17(b). We can choose one conformality factor at arbitrary vertex of each independent $T$-cycle as a free variable(such as $d_{1}$ and $d_{13}$ ) and the dimension of $S\left(2,2,1,1, \mathscr{T}_{P}\right)$ is 37 .

For example, if $\left(x_{0}, x_{1}, \cdots, x_{11}\right)=\left(0,1,2, \frac{7}{3}, \frac{8}{3}, 3, \frac{11}{3}, 4, \frac{13}{3}, \frac{14}{3}, \frac{17}{3}, 6\right)$ and $\left(y_{0}, y_{1}, \cdots\right.$, $\left.y_{11}\right)=\left(0,1,2, \frac{7}{3}, \frac{8}{3}, 3, \frac{10}{3}, \frac{11}{3}, 4, \frac{13}{3}, \frac{16}{3}, 6\right)$, then $a_{1}=a_{2}=1$ in this case.

Case 2: If $a_{1}=1$ and $a_{2} \neq 1, d_{6}$ is determined by $d_{20}, d_{13}$ is determined by $d_{6}$ (or $d_{20}$ ) by (C.1) and (C.2), then $d_{2}$ and $d_{6}$ (or $d_{2}$ and $d_{20}$ ) are two free variables, the structure is non-degenerate and the dimension of $S\left(2,2,1,1, \mathscr{T}_{P}\right)$ is 37 ;

Case 3: If $a_{1} \neq 1$ and $a_{2}=1, d_{6}$ is determined by $d_{20}, d_{2}$ is determined by $d_{6}$ (or $d_{20}$ ) by (C.1) and (C.2), then $d_{13}$ and $d_{6}$ (or $d_{13}$ and $d_{20}$ ) are two free variables, the structure is non-degenerate and the dimension of $S\left(2,2,1,1, \mathscr{T}_{P}\right)$ is 37 ;

Case 4: If $a_{1} \neq 1$ and $a_{2} \neq 1, d_{6}$ and $d_{20}$ are free variables, the structure is nondegenerate and the dimension of $S\left(2,2,1,1, \mathscr{T}_{P}\right)$ is 37 .

The proof is completed. 
Acknowledgments This work is supported by the National Natural Science Foundation of China (Nos. 11471066, 11572081, 11290143, 11601064), Fundamental Research of Civil Aircraft (No. MJ-F-2012-04), and the Fundamental Research Funds for the Central Universities (DUT15LK44).

\section{References}

[1] T. W. Sederberg, J. Zheng, A. Bakenov and A. Nasri, T-splines and T-NURCCs, ACM Trans. Graph., 22 (2003), pp. 477-484.

[2] T. W. Sederberg, D. L. Cardon, G. T. Finnigan, N. S. North, J. Zheng and T. Lyche, T-spline simplification and local refinement, ACM Trans. Graph., 23 (2004), pp. 276-283.

[3] X. Li, J. Zheng, T. W. Sederberg, T. J. R. Hughes And M. A. Scott, On the linear independence of T-splines blending functions, Comput. Aided Geom. Des., 29 (2012), pp. 63-76.

[4] M. A. Scott, X. Li, T. W. Sederberg And T. J. R. Hughes, Local refinement of analysis-suitable T-splines, Comput. Methods Appl. Mech. Eng., 213 (2012), pp. 206-222.

[5] J. S. Deng, F. L. Chen And Y. Y. Feng, Dimensions of spline spaces over T-meshes, J. Comput. Appl. Math., 194 (2006), pp. 267-283.

[6] Z. J. Huang, J. S. Deng, Y. Y. FEng And F. L. Chen, New proof of dimension formula of spline spaces over T-meshes via smoothing cofactors, J. Comput. Math., 24 (2006), pp. 501-514.

[7] R. H. WANG, The structural characterization and interpolation for multivariate splines, Acta Math. Sinica, 18 (1975), pp. 91-106.

[8] R. H. WAnG, Multivariate Spline Functions and Their Applications, Science Press/Kluwer Pub., Beijing/New York, 2001.

[9] C. J. LI, R. H. WANG AND F. ZhANG, Improvement on the dimension of spline spaces on T-mesh, J. Inf. Comput. Sci., 3 (2006), pp. 235-244.

[10] R. H. WANG, C. J. Li AND J. Chen, The dimensions of spline spaces on Quasi-rectangular meshes, J. Math. Res. Exposition, 28 (2008), pp. 745-752.

[11] C. J. Li AND J. Chen, On the dimensions of bivariate spline spaces and the stability of the dimensions, J. Comput. Appl. Math., 236 (2011), pp. 765-774.

[12] B. Mourrain, On the dimension of spline spaces on planar T-meshes, Math. Comp., 83 (2014), pp. 847-871.

[13] X. Li And J. S. Deng, On the dimension of spline spaces over T-meshes with smoothing cofactorconformality method, Comput. Aided Geom. Des., 41 (2016), pp. 76-86.

[14] Z. H. WANG, F. L. Chen AND J. S. Deng, Evaluation algorithm of PHT-spline surfaces, Numer. Math. Theor. Meth. Appl., 10 (2017), pp. 760-774.

[15] J. Morgan And R. ScotT, The dimension of $C^{1}$ piecewise polynomials, Unpublished manuscript, 1977.

[16] X. Li And F. L. Chen, On the instability in the dimension of spline space over particular T-meshes, Comput. Aided Geom. Des., 28 (2011), pp. 420-426.

[17] D. Berdinsky, M. Оh, T. Kim And B. Mourrain, On the problem of instability in the dimension of a spline space over a T-mesh, Comput. Graph., 36 (2012), pp. 507-513.

[18] Q. J. Guo, R. H. WAng And C. J. Li, On the problem of instability in the dimension of spline spaces over T-meshes with T-cycles, J. Comput. Math., 33 (2015), pp. 248-262.

[19] X. Q. SHI, The singularity of Morgan-Scott triangulation, Comput. Aided Geom. Des., 8 (1991), pp. 201-206.

[20] D. DiEnER, Instability in the dimension of spaces of bivariate piecewise polynomials of degree $2 r$ and smoothness order $r$, SIAM J. Numer. Anal., 2 (1990), pp. 543-551. 
[21] H. Du, X. Q. Shi And R. H. Wang, A geometric approach to dim $S_{2}^{1}\left(\Delta_{M S}\right)$, J. Syst. Sci. Complex., 15 (2002), pp. 92-94.

[22] Z. X. Luo, Generator basis of module in $K[X]^{m}$ and its applications, Acta Math. Sinica, 44 (2001), pp. 983-994. 